\title{
ONE-DIMENSIONAL FIBONACCI QUASILATTICES AND THEIR APPLICATION TO THE EUCLIDEAN ALGORITHM AND DIOPHANTINE EQUATIONS
}

\author{
V. G. ZHURAVLEV
}

\begin{abstract}
The one-dimensional quasilattices $\mathcal{L}$ lying in the square Fibonacci quasilattice $\mathcal{F}^{2}=\mathcal{F} \times \mathcal{F}$ are classified; here $\mathcal{F}$ is the one-dimensional Fibonacci quasilattice. It is proved that there exists a countable set of similarity classes of quasilattices $\mathcal{L}$ in $\mathcal{F}^{2}$ (fine classification), and also four classes of local equivalence (rough classification).

Asymptotic distributions of points in quasilattices $\mathcal{L}$ are found and then applied to Diophantine equations involving the function $[\alpha]$ (the integral part of $\alpha$ ) and to equations of the form $A_{1} \circ X_{1}-A_{2} \circ X_{2}=C$, where the coefficients $C$ and $A_{i}$ and the variables $X_{i}$ take values in $\mathbb{N}=\{1,2,3, \ldots\}$ and $\circ$ is Knuth's circular multiplication.
\end{abstract}

\section{INTRODUCTION}

0.1. The one-dimensional Fibonacci quasilattice $\mathcal{F}$ is the subset of $\mathbb{R}$ consisting of the points $x=\delta(N)=N+[(N+1) \tau] \cdot \tau$, where $N \in \mathbb{Z},[*]$ is the integral part function, and $\tau=(-1+\sqrt{5}) / 2$ is the golden section. The quasilattice $\mathcal{F}$ is not periodic, i.e., the relation $\mathcal{F}+t=\mathcal{F}$ is valid only if $t=0$. A natural approach to finding other quasilattices similar to $\mathcal{F}$ is to introduce the square Fibonacci quasilattice $\mathcal{F}^{2}=\mathcal{F} \times \mathcal{F}$ (see Figure 1.1). With the help of the "cut and project" method (see, e.g., [10]), the quasilattice $\mathcal{F}^{2}$ can be characterized by the equation

$$
\left(\mathcal{F}^{2}\right)^{\prime}=\mathcal{O}^{2} \cap J^{2},
$$

where $\mathcal{O}$ is the ring of integers in the quadratic field $F=\mathbb{Q}(\tau)$, ' denotes conjugation in $F$, and $J=[-1, \tau)$. In (0.1), $J^{2}=J \times J$ is a "window", or an interior space, which distinguishes $\mathcal{F}^{2}$ in the dense set $\mathcal{O}^{2}$ as an $(r, R)$-Delone system, i.e., a not too dense and not too rarefied system of points in the plane $\mathbb{R}^{2}$.

The two-dimensional quasilattices $\mathcal{F}^{2}$ were introduced and studied for the first time by Lifshitz in [7]. In the present paper, we use $\mathcal{F}^{2}$ as a two-dimensional quasilattice containing all basic types of the one-dimensional Fibonacci quasilattices, which makes it possible to obtain a complete classification of the latter.

0.2. We choose two arbitrary points in $\mathcal{F}^{2}$ and consider the line $L$ passing through these points. Then $L$ gives rise to a one-dimensional quasilattice $\mathcal{L}$ embedded in $\mathcal{F}^{2}$,

$$
L \rightarrow \mathcal{L}=L \cap \mathcal{F}^{2} .
$$

\footnotetext{
2000 Mathematics Subject Classification. Primary 06A11.
}

Key words and phrases. Fibonacci quasilattices, Diophantine equations, Knuth's circular multiplication.

Supported by RFBR (grant no. 05-01-00435). 
In this paper, we classify all quasilattices $\mathcal{L}$ in $\mathcal{F}^{2}$ that arise in this way. This classification is based on the parametrization (see Lemma 3.1)

$$
\Gamma \stackrel{\sigma}{\sim} \mathcal{L}: \quad t^{\prime} \mapsto x=\sigma\left(t^{\prime}\right)=t \cdot \beta+v
$$

of the quasilattice $\mathcal{L}=\mathcal{L}(\beta, v, \gamma)$ by points $t^{\prime}$ of the parameter set $\Gamma=\gamma \cap \mathcal{O}$, where $\gamma$ is an interval in $J, \beta=\left(\beta_{1}, \beta_{2}\right)$ is a primitive direction vector of $L$ (0.2), and $v$ is a shift vector.

0.3. Two one-dimensional quasilattices $\mathcal{L}=\mathcal{L}(\beta, v, \gamma)$ and $\mathcal{L}^{\prime}=\mathcal{L}^{\prime}\left(\beta^{\prime}, v^{\prime}, \gamma^{\prime}\right)$ are said to be similar (we write $\mathcal{L} \stackrel{s}{\sim} \mathcal{L}^{\prime}$ ) if there is a similarity transformation of $\mathbb{R}^{2}$ that takes one quasilattice to the other. In Theorem 6.1, it is proved that two quasilattices $\mathcal{L}$ and $\mathcal{L}^{\prime}$ in the square quasilattice $\mathcal{F}^{2}$ are similar if and only if

$$
|\gamma| \sim\left|\gamma^{\prime}\right| \bmod E_{+},
$$

which means that $|\gamma| /\left|\gamma^{\prime}\right| \in E_{+}$, where $E_{+}=\left\{\tau^{m} ; m \in \mathbb{Z}\right\}$ is the subgroup of positive units in the group of units $\mathcal{O}^{\times}$of the Fibonacci ring $\mathcal{O}$. Using the parametrization (0.3), we can prove that the set of similarity classes of quasilattices is in one-to-one correspondence with the numbers in the set $[\tau, 1) \cap \mathbb{Q}(\tau)$.

0.4. Two one-dimensional quasilattices $\mathcal{L}$ and $\mathcal{L}^{\prime}$ in $\mathcal{F}^{2}$ are of the same local type (or are locally equivalent), $\mathcal{L} \stackrel{l}{\sim} \mathcal{L}^{\prime}$, if there is a similarity transformation $s$ such that the quasilattices $\mathcal{L}$ and $s \mathcal{L}^{\prime}$ have one and the same set of distances between neighboring points. This set can contain either two distances $g, e$, or three distances $g, e, g+e$, where $g / e=\tau$. Let $x$ be an arbitrary point of $\mathcal{L}$, and let $x_{-}, x_{+} \in \mathcal{L}$ be points neighboring to $x$ and ordered in accordance with the direction vector $\beta$. Then there is a point $x^{\prime} \in \mathcal{L}^{\prime}$ for which the neighboring distances are $\left|x_{-}^{\prime}-x^{\prime}\right|=\left|x_{-}-x\right|$ and $\left|x_{+}^{\prime}-x^{\prime}\right|=\left|x_{+}-x\right|$, and vice versa. The definition implies that locally equivalent quasilattices $\mathcal{L}$ and $\mathcal{L}^{\prime}$ are locally indistinguishable.

In Table 0.1 all possible first neighborhoods of points are given for the quasilattices $\mathcal{L}$ of local types $j=0,1,2,3$. Let, for example, $j=0$, and let $x_{-}, x_{+} \in \mathcal{L}$ be the neighboring points for $x \in \mathcal{L}$. Then the distances $r_{\mp}=\left|x_{\mp}-x\right|$ can take the following three values: $\left(r_{-}, r_{+}\right)=(g, e),(e, g)$, or $(e, e)$. Table 0.1 shows how various types of local neighborhoods arise or disappear as the length of the interval $\gamma$ for the quasilattice $\mathcal{L}=$ $\mathcal{L}(\beta, v, \gamma)$ changes. The number of all local neighborhoods is shown in the second column. The local types $j=0,1,2,3$ are distinct, and the next local type $j=0^{\prime}$ is equivalent to $j=0$ (see Figure 6.1). Passage from one level to another implies multiplication of the local distances by $\tau+1$, which yields the periodicity $j \bmod 4$.

In Theorem 6.2, we prove that each one-dimensional quasilattice $\mathcal{L}$ in the two-dimensional Fibonacci quasilattice $\mathcal{F}^{2}$ has one of the local types $j$, where $j=0,1,2,3$, and each local type is realized by a quasilattice $\mathcal{L}$ in $\mathcal{F}^{2}$.

TABLE 0.1. Local types of one-dimensional quasilattices $\mathcal{L}$ in $\mathcal{F}^{2}$

\begin{tabular}{|l|l|l|l|l|l|}
\hline$j=0$ & 3 & $(g, e),(e, g)$ & $(e, e)$ & & \\
\hline$j=1$ & 5 & $(g, e),(e, g)$ & $(e, e)$ & $(e, g+e),(g+e, e)$ & \\
\hline$j=2$ & 4 & $(g, e),(e, g)$ & & $(e, g+e),(g+e, e)$ & \\
\hline$j=3$ & 5 & $(g, e),(e, g)$ & & $(e, g+e),(g+e, e)$ & $(g+e, g+e)$ \\
\hline$j=0^{\prime}$ & 3 & & & $(e, g+e),(g+e, e)$ & $(g+e, g+e)$ \\
\hline
\end{tabular}


Every two quasilattices of the same dimension over $\mathbb{Z}$ are similar. This fact is in contrast to the existence of countably many similarity classes of one-dimensional quasilattices $\mathcal{L}$ in $\mathcal{F}^{2}$. Moreover, even on the local level, there are four distinct types of such quasilattices. They all form a class of one-dimensional Fibonacci quasilattices that are characterized as quasilattices parametrized by circle rotations and the induced mappings (Poincaré mappings).

0.5. The level $m=0,1,2, \ldots$ of a quasilattice $\mathcal{L}=\mathcal{L}(\beta, v, \gamma)$ (0.3) is defined by the relations

$$
\tau^{m}<|\gamma|<\tau^{m-1} \quad \text { or } \quad|\gamma|=\tau^{m-1},
$$

and the lattice $\mathcal{L}$ is said to be nondegenerate or degenerate, respectively. In the first case, the interval $\gamma$ is split into half-open intervals,

$$
\gamma=\gamma_{G} \oplus \gamma_{G E} \oplus \gamma_{E},
$$

and the bijection $\sigma$ (see (0.3)) is a parametrization of the quasilattices $\mathcal{L}$ by noncyclic rearrangements of the half-open intervals in (0.6). In the second case in (0.5), the halfopen interval $\gamma_{G E}$ in (0.6) disappears. Therefore, the quasilattices $\mathcal{L}$ are parametrized by rearrangements of two half-open intervals or, which is the same, by circle rotations (see Theorem 4.1).

We split the set of points of the quasilattice $\mathcal{L}=\mathcal{L}_{G} \cap \mathcal{L}_{G E} \cap \mathcal{L}_{E}$ and its parameter set $\Gamma=\Gamma_{G} \cap \Gamma_{G E} \cap \Gamma_{E}$ in accordance with (0.6). We assume that $\beta_{1} \neq 0$ for the direction vector $\beta=\left(\beta_{1}, \beta_{2}\right)$, and denote by $\operatorname{pr}_{1} x=x_{1}$ the projection of the vector $x=\left(x_{1}, x_{2}\right)$ to the axis $O X$. For each type of points $x=x_{Y}$ in $\mathcal{L}$, where $Y$ is $G, G E$, or $E$, we define the frequency

$$
p_{Y}=\lim _{X \rightarrow \infty} \frac{\sharp\left(\operatorname{pr}_{1} \mathcal{L}_{Y} \cap\left[X_{1}, X_{1}+X\right]\right)}{\sharp\left(\operatorname{pr}_{1} \mathcal{L} \cap\left[X_{1}, X_{1}+X\right]\right)}
$$

of its occurrence in the quasilattice $\mathcal{L}=\mathcal{L}(\beta, v, \gamma)$. If the level $m$ of the quasilattice is even, then, by Theorem 5.1, the frequencies $p_{Y}$ of its points $x_{Y} \in \mathcal{L}$ are calculated by the formulas

$$
p_{G}=1-\frac{\tau^{m}}{|\gamma|}, \quad p_{G E}=\frac{\tau^{m-1}}{|\gamma|}-1, \quad p_{E}=1-\frac{\tau^{m+1}}{|\gamma|}
$$

if $\mathcal{L}$ is a nondegenerate lattice, and by the formulas

$$
p_{G}=\tau, \quad p_{G E}=0, \quad p_{E}=\tau^{2}
$$

if $\mathcal{L}$ is degenerate. Similar formulas are valid if the level $m$ is odd. Let $n_{\mathcal{L}}(X)$ be the number of points $x=\left(x_{1}, x_{2}\right)$ with $0 \leq x_{1} \leq X$ in the quasilattice $\mathcal{L}$. Then $n_{\mathcal{L}}(X)$ satisfies the following asymptotic relation:

$$
n_{\mathcal{L}}(X)=k X+o(X) \text { for } X \rightarrow+\infty,
$$

where $k=\frac{|\gamma|}{(1+2 \tau)\left|\beta_{1}\right|}$.

0.6. Formula (0.9) has two applications to Diophantine equations. We consider a system of Diophantine equations

$$
a_{1} N_{1}+a_{2} N_{2}=c_{1}, \quad a_{1}\left[N_{1} \tau\right]+a_{2}\left[N_{2} \tau\right]=c_{2},
$$

where the coefficients $a_{i}, c_{i}$ and the variables $N_{i}$ belong to the ring $\mathbb{Z}$ of rational integers. We assume that the coefficients satisfy the following conditions: $a_{1}$ and $a_{2}$ are relatively prime, $a_{2} \geq-a_{1} \geq 3, c^{\prime}>0, m<c^{\prime}<M$, and $-1<\frac{c^{\prime}-a_{2} \tau}{a_{1}}<\tau$, where $c^{\prime}=$ $c_{1}-c_{2} \widetilde{\tau}-a_{1}-a_{2}$, and $m$ and $M$ are, respectively, the minimal and the maximal number in the set $\left\{a_{1} x_{1}+a_{2} x_{2} ; x_{1}=\tau,-1, x_{2}=\tau,-1\right\}$. Then system (0.10) has a solution, 
and the number $n(X)$ of its solutions $\left(N_{1}, N_{2}\right)$ with $X_{1} \leq N_{1} \leq X_{1}+X$, where $X_{1}$ is fixed and $X \rightarrow+\infty$, satisfies the asymptotic identity

$$
n(X)=k X+o(X), \quad \text { where } k=\frac{\left|a_{2}-c_{1} \tau+c_{2}\right|}{\left|a_{1}\right| a_{2}^{2}}
$$

(see Proposition 5.1).

The second application deals with the equation

$$
A_{1} \circ X_{1}-A_{2} \circ X_{2}=C,
$$

where the coefficients $A_{i}, C$ and the variables $X_{i}$ take values in $\mathbb{N}=\{1,2,3, \ldots\}$ and $\circ$ is Knuth's circular multiplication [6]. Let $\left|\delta^{\prime}\left(A_{i}\right)\right| \leq \tau^{3}$, and let $n_{F / \mathbb{Q}}\left(\delta\left(A_{i}\right)\right)(i=1,2)$ be relatively prime, where $n_{F / \mathbb{Q}}(\delta(A))=A^{2}-A[(A+1) \tau]-[(A+1) \tau]^{2}$ is the norm of $\delta(A)=$ $A+[(A+1) \tau] \tau$ in the quadratic extension $F / \mathbb{Q}$. We assume that one of the following conditions 1) or 2) is valid: 1) $0<\frac{\delta^{\prime}\left(A_{2}\right)}{\delta^{\prime}\left(A_{1}\right)}<1$ and $m<\delta^{\prime}(C)<M$, where $m$ is the minimal and $M$ is the maximal number in the set $\left\{\delta^{\prime}\left(A_{1}\right) \tau-\delta^{\prime}\left(A_{2}\right) \tau,-\delta^{\prime}\left(A_{1}\right)+\delta^{\prime}\left(A_{2}\right)\right\}$; 2) $-\tau<\frac{\delta^{\prime}\left(A_{2}\right)}{\delta^{\prime}\left(A_{1}\right)}<0$ and $m<\delta^{\prime}(C)<M$, where $m$ is the minimal and $M$ is the maximal number in the set $\left\{\delta^{\prime}\left(A_{1}\right) \tau+\delta^{\prime}\left(A_{2}\right),-\delta^{\prime}\left(A_{1}\right)-\delta^{\prime}\left(A_{2}\right) \tau\right\}$. Under these conditions, the number $n(X)$ of solutions $\left(X_{1}, X_{2}\right)$ of equation (0.12) with $1 \leq X_{2} \leq X$ is calculated, as $X \rightarrow+\infty$, by the asymptotic formula

$$
n(X)=k X+o(X), \quad \text { where } k=\frac{1}{\left|n_{F / \mathbb{Q}}\left(\delta\left(A_{1}\right)\right)\right|} .
$$

Formulas (0.11) and (0.13) show that the Diophantine equations (0.10) and (0.12) are sensitive to the metric characteristics of their coefficients. Therefore, it is natural to state general results concerning Diophantine equations of this type in geometric terms, similar to what was done in the asymptotic formula (0.9).

Earlier in [3], the quasilattice $\mathcal{F}^{2}$ was applied to quadratic Diophantine equations.

0.7. The Fibonacci ring $\mathcal{O}=\mathbb{Z}[\tau]$ is Euclidean. It admits a division algorithm with respect to the norm (see [1]). Yet another application of one-dimensional quasilattices is that the normalized quasilattice $\mathcal{F}_{1}=\tau \cdot \mathcal{F}$ determines, in the terminology of Cooke [4], a one-dimensional two-step Euclidean algorithm in which the maximal number of the form

$$
\delta_{1}(N)=[(N+1) \tau]+(N-[(N+1) \tau]) \cdot \tau \leq \alpha / \beta
$$

with $N=0,1,2, \ldots$ is taken as the quotient for $\alpha / \beta$, where $\alpha, \beta \in \mathcal{O}$ and $\alpha, \beta>0$. In Theorem 7.1, we obtain an upper bound for the number of steps in the algorithm, which shows that the one-dimensional algorithm in question is fast.

\section{§1. FibonacCi QUasilattices}

1.1. The one-dimensional Fibonacci quasilattice $\mathcal{F}$. We introduce the mapping

$$
\mathbb{Z} \stackrel{\delta^{\prime}}{\longrightarrow} \mathbb{R}: \quad N \mapsto \delta^{\prime}(N)=N-r(N) \widetilde{\tau}
$$

such that $\delta^{\prime}(\mathbb{N}) \subset J$ and $\overline{\delta^{\prime}(\mathbb{N})}=\bar{J}$; here $\widetilde{\tau}=\tau+1$, the function $r(N)$ takes values in $\mathbb{N}=\{0,1,2, \ldots\}, J=[-1, \tau)$, and the bar stands for closure. Then $r(N)=[(N+1) \tau]$ (see [3]), where $[*]$ is the integral part function, and we can represent the mapping (1.1) by the explicit formula

$$
\delta^{\prime}(N)=N-[(N+1) \tau] \widetilde{\tau} .
$$

The one-dimensional Fibonacci quasilattice $\mathcal{F}$ is the discrete subset of $\mathbb{R}$ defined by (see Figure 1.1)

$$
\mathcal{F}=\delta(\mathbb{Z})
$$


where $\delta(N)=N+r(N) \tau$ for all $N \in \mathbb{Z}$. We note that $\delta(N)=\delta^{\prime}(N)^{\prime}$, where ' denotes conjugation in the quadratic field $F=\mathbb{Q}(\sqrt{5})$. The quasilattice $\mathcal{F}$ has many interesting properties. For example, in $\S 7$ we shall apply the construction of the division algorithm in the ring $\mathcal{O}$ to $\mathcal{F}$. Here, we list some simple properties of $\mathcal{F}$.

We denote by $\mathcal{F}^{+}$the subset of $\mathcal{F}$ consisting of all points of the form $\delta(N)$ with $N \in \mathbb{N}$. For the difference function $\Delta r(N)=r(N)-r(N-1)$, we have $\Delta r(N)=1$ if $N \tau \bmod 1 \in\left[\tau^{2}, 1\right)$ and $\Delta r(N)=0$ if $N \tau \bmod 1 \in\left[0, \tau^{2}\right)$. This shows that the set $\mathcal{F}^{+}$ is parametrized by circle rotations. The results of [5] imply that

$$
u=\left(u_{1}, u_{2}, \ldots, u_{N}, \ldots\right)=(10110101 \ldots),
$$

where $u_{N}=\Delta r(N)=0,1$, is a Sturm sequence. The sequence $u$ completely determines the positive part $\mathcal{F}^{+}$of the quasilattice $\mathcal{F}$, because $\Delta(\delta(N))=1+\tau$ if $u_{N}=1$ and $\Delta(\delta(N))=1$ if $u_{N}=0$. The sequence $u$ is invariant under the substitution that acts on the symbols 0,1 by the rule $\pi: 1 \mapsto 10, \quad 0 \mapsto 1$, and is obtained from 1 by repeated application of $\pi$. The length of a sequence $\pi^{n}(1)$ is equal to $\sharp \pi^{n}(1)=F_{n+1}$, where the $F_{m}$ are the Fibonacci numbers defined by the recurrence relation $F_{m+2}=F_{m+1}+F_{m}$ and the initial condition $F_{1}=1, F_{2}=2$. For the sequences $\pi^{m}(1)$, we also have the recurrence relation

$$
\pi^{n+1}(1)+\pi^{n}(1)=\pi^{n+2}(1)
$$

where, on the left-hand side, we have the sequence obtained by successive extension of $\pi^{n+1}(1)$ by elements of the sequence $\pi^{n}(1)$ (this is, by a noncommutative operation of juxtaposition). For example, $\pi^{2}(1)+\pi^{1}(1)=(101)+(10)=(10110)$. We put $\mathcal{F}_{N}^{+}=$ $\left\{\delta(M) \in \mathcal{F}^{+}: M=0,1, \ldots, N-1\right\}$. Then (1.5) implies the relation

$$
\mathcal{F}_{F_{n+1}}^{+} \cup\left(\mathcal{F}_{F_{n}}^{+}+\delta\left(F_{n+1}\right)\right)=\mathcal{F}_{F_{n+2}}^{+}, \quad n=1,2,3, \ldots,
$$

which is a recurrence property of finite intervals of the sublattice $\mathcal{F}^{+} \subset \mathcal{F}$. Thus, $\mathcal{F}^{+}$ can be obtained by the operations of addition and passage to the limit from its two parts $\mathcal{F}_{F_{1}}^{+}=\{\delta(0)\}=\{0\}$ and $\mathcal{F}_{F_{2}}^{+}=\{\delta(0), \delta(1)\}=\{0,1+\tau\}$.

The set $\delta^{\prime}(\mathbb{Z}) \backslash\left\{\delta^{\prime}(-1)\right\}$ is centrally symmetric: $\iota \delta^{\prime}(N)=-\delta^{\prime}(N)+\delta^{\prime}(-2)$ for every $N \in \mathbb{Z} \backslash\{-1\}$. Using formula (1.2), we can represent $\iota$ in terms of the parameters, $\iota \delta^{\prime}(N)=\delta^{\prime}(-N-2)$. It follows that the punctured quasilattice $\mathcal{F}_{\text {hole }}=\mathcal{F} \backslash\{\delta(-1)\}=$ $\mathcal{F} \backslash\{-1\}$ is centrally symmetric,

$$
\iota \mathcal{F}_{\text {hole }}=\mathcal{F}_{\text {hole }},
$$

with the center of symmetry $\frac{1}{2} \delta(-2)=\frac{-1+\tau}{2}$, which is not an element of $\mathcal{F}_{\text {hole }}$.

Apart from the symmetry $\iota$, the quasilattice $\mathcal{F}$ has a rich semigroup of similarities $\delta\left(\mathbb{Z}_{\tau}\right) \subset \mathcal{F}$, where $\mathbb{Z}_{\tau}$ is the subset of all $N \in \mathbb{Z}$ for which $\left|\delta^{\prime}(N)\right|<\tau$. For all $N \in \mathbb{Z}_{\tau}$, we have

$$
\delta(N) \cdot \mathcal{F} \subset \mathcal{F} \text {. }
$$

Of special interest is the case of $N=1$, where we have a similarity

$$
\lambda \cdot \mathcal{F} \subset \mathcal{F}
$$

the coefficient $\lambda=\delta(1)=\widetilde{\tau}$ of which is a fundamental unit of the Fibonacci ring $\mathcal{O}=$ $\mathbb{Z}\left[\widetilde{\tau}\right.$. In this case, the similarity (1.7) is the inflation of $\mathcal{F}_{\text {hole }}$ corresponding to the substitution $\pi$.

The quasilattice $\mathcal{F}$ is not periodic; i.e., the relation $\mathcal{F}+t=\mathcal{F}$ is valid only for $t=0$. This follows from a similar property of the parameter set $\delta^{\prime}(\mathbb{Z})$. However, we have

$$
\mathcal{F}+\mathcal{F} \subset \mathcal{F}+\mathcal{R}, \quad \mathcal{F}-\mathcal{F} \subset \mathcal{F}+\mathcal{R},
$$

where $\mathcal{R}=\{0, \pm \tau\}$, which means that the quasilattice $\mathcal{F}$ is "almost" closed under the operations of addition and subtraction (the Meier property). 


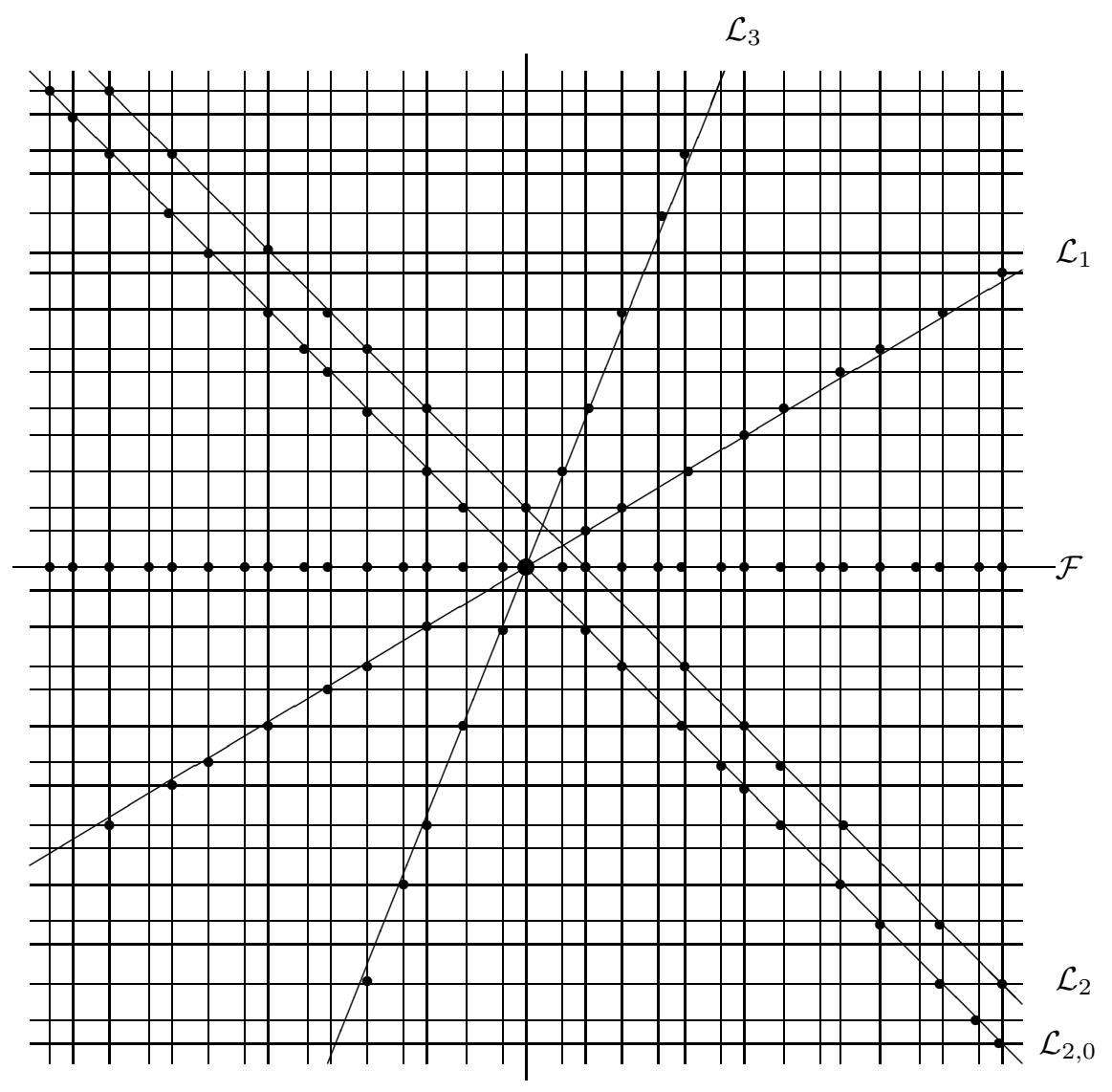

Figure 1.1. Quadratic Fibonacci quasilattice $\mathcal{F}^{2}$

1.2. The quadratic Fibonacci quasilattice $\mathcal{F}^{2}$. To extend the class of one-dimensional quasilattices, we introduce the square quasilattice (see Figure 1.1)

$$
\mathcal{F}^{2}=\mathcal{F} \times \mathcal{F}=\left\{\left(x_{1}, x_{2}\right)=\left(\delta\left(N_{1}\right), \delta\left(N_{1}\right)\right) ; N_{i} \in \mathbb{Z}\right\} .
$$

This quasilattice can be characterized as the set of all points $\left(x_{1}, x_{2}\right)$ in $\mathcal{O}^{2}$ such that $\left(x_{1}^{\prime}, x_{2}^{\prime}\right) \in J^{2}$, or more concisely,

$$
\left(\mathcal{F}^{2}\right)^{\prime}=\mathcal{O}^{2} \cap J^{2} .
$$

Let $L$ be a line passing through two points of $\mathcal{F}^{2}$. Then $\mathcal{L}=L \cap \mathcal{F}^{2}$ is a one-dimensional quasilattice. In particular, if $L$ is the main diagonal, then we obtain a quasilattice $\mathcal{L}$ similar to $\mathcal{F}$. If $L$ is the second diagonal, then $\mathcal{L}$ is no longer similar to $\mathcal{F}$, because the distances between neighboring points in $\mathcal{L}$ take three distinct values (see the quasilattice $\mathcal{L}_{2,0}$ in Figure 1.1. .

To classify the one-dimensional lattices $\mathcal{L}$ arising in this way, we need to study in detail the Fibonacci tilings of the half-open interval $J$.

In essence, we use the well-known "cut and project" method (see, e.g., [10]) and choose the square $J^{2}$ for the role of the "window" or the interior space $W$ in the definition (1.10). If an arbitrary convex set is chosen for $W$, then no new types of quasilattices $\mathcal{L}$ arise. If we admit nonconvex $W$, then new quasilattices $\mathcal{L}$ appear. They are unions of old quasilattices. 


\section{§2. One-dimensional Fibonacci QuAsilattices AND IndUCED MAPPings}

2.1. Proper Fibonacci tilings. We identify the half-open interval $I=[0,1)$ with the unit circle $\mathbb{R} / \mathbb{Z}$ and define the shift

$$
S: x \mapsto x+\tau \bmod 1,
$$

where $\tau=\frac{-1+\sqrt{5}}{2}$ is the golden section. Since 1 and the shift $\tau$ are incommensurable, we can define the following induced mapping (the Poincaré mapping) for every $\varepsilon \in I$ :

$$
S_{\varepsilon}=\left.S\right|_{I(\varepsilon)}: I(\varepsilon) \rightarrow I(\varepsilon),
$$

where $I(\varepsilon)=[\varepsilon, 1)$ is the interval on which $S_{\varepsilon}$ acts. It is well known [2] that the induced mapping $S_{\varepsilon}$ is isomorphic either to 1) a noncyclic rearrangement of three half-open intervals in $I(\varepsilon)$, or to 2 ) a rearrangement of two half-open intervals in $I(\varepsilon)$, which is equivalent to a rotation of the unit circle. Case 2) arises when the parameter $\varepsilon$ takes the values

$$
\varepsilon_{m}=1-\tau^{m} \quad \text { for } m=0,1,2, \ldots .
$$

For such an $\varepsilon$, the half-open interval $I_{m}=I\left(\varepsilon_{m}\right)=\left[\varepsilon_{m}, 1\right)$ is split into two half-open intervals $G_{0}^{m}$ and $E_{0}^{m}$,

$$
I_{m}=G_{0}^{m} \oplus E_{0}^{m},
$$

for which the ratio of lengths is $\left|G_{0}^{m}\right| /\left|E_{0}^{m}\right|=\tau$ or $\tau^{-1}$ for $m$ even or odd, respectively. In (2.3), $\oplus$ means the noncommutative operation of juxtaposition of intervals. The induced mapping $S^{(m)}=S_{\varepsilon_{m}}$ interchanges (see [2]) the half-open intervals of the partition (2.3),

$$
S^{(m)}: G_{0}^{m} \oplus E_{0}^{m} \rightarrow E_{0}^{m} \oplus G_{0}^{m} .
$$

Here, the induced mapping $S^{(m)}$ is isomorphic to the shift $S$ or to the inverse shift $S^{-1}$, depending on whether $m$ is even or odd,

$$
S^{(m)} \sim S^{ \pm 1}
$$

We obtain a proper Fibonacci tiling $\operatorname{Til}\left(\varepsilon_{m}\right)$ of level $m$ of the unit half-open interval $I$ if we apply the shift $S$ several times to the half-open intervals $G_{0}^{m}, E_{0}^{m} \subset I_{m} \subset I$ until they return into $I_{m}$ for the first time. The tiling Til $\left(\varepsilon_{m}\right)$ consists of $F_{m+1}$ and $F_{m+2}$ half-open intervals $\left|G_{0}^{m}\right|$ and $\left|E_{0}^{m}\right|$, respectively, if the level $m$ is even, and $F_{m+2}$ and $F_{m+1}$ half-open intervals if $m$ is odd. In [2], a structure theorem concerning the invariance of the tiling $\operatorname{Til}\left(\varepsilon_{m}\right)$ under the shift $S$ was proved,

$$
S\left(\operatorname{Til}\left(\varepsilon_{m}\right)\right)=\operatorname{Til}^{+}\left(\varepsilon_{m}\right),
$$

where the tiling $\mathrm{Til}^{+}\left(\varepsilon_{m}\right)$ is obtained by a rearrangement of the two rightmost half-open intervals $G_{0}^{m}$ and $E_{0}^{m}$ in $\operatorname{Til}\left(\varepsilon_{m}\right)$ (see (2.4)).

2.2. Improper Fibonacci tilings. An improper tiling $\operatorname{Til}(\varepsilon)$, where $\varepsilon_{m} \leq \varepsilon<\varepsilon_{m+1}$ and $m \geq 0$, is obtained from $\operatorname{Til}\left(\varepsilon_{m}\right)$ by dividing each of the half-open intervals in $\operatorname{Til}\left(\varepsilon_{m}\right)$ into two half-open intervals so that the leftmost interval will be of length $\delta=\varepsilon-\varepsilon_{m}$. The half-open interval $I(\varepsilon)$ of length $1-\varepsilon$ will split into three half-open intervals,

$$
I(\varepsilon)=G_{0}^{m}(\varepsilon) \oplus G E_{0}^{m}(\varepsilon) \oplus E_{0}^{m}(\varepsilon),
$$

which have lengths

$$
\left|G_{0}^{m}(\varepsilon)\right|=\tau^{m+2}-\delta, \quad\left|G E_{0}^{m}(\varepsilon)\right|=\delta, \quad\left|E_{0}^{m}(\varepsilon)\right|=\tau^{m+1}-\delta
$$

if $m$ is even, and

$$
\left|G_{0}^{m}(\varepsilon)\right|=\tau^{m+1}-\delta, \quad\left|G E_{0}^{m}(\varepsilon)\right|=\delta, \quad\left|E_{0}^{m}(\varepsilon)\right|=\tau^{m+2}-\delta
$$


if $m$ is odd. As was noted in Subsection 2.1, the induced mapping $S_{\varepsilon}$ rearranges the half-open intervals of the partition (2.7) noncyclically,

$$
S_{\varepsilon}\left(G_{0}^{m}(\varepsilon) \oplus G E_{0}^{m}(\varepsilon) \oplus E_{0}^{m}(\varepsilon)\right)=E_{0}^{m}(\varepsilon) \oplus G E_{0}^{m}(\varepsilon) \oplus G_{0}^{m}(\varepsilon) .
$$

Consequently, the induced mapping $S_{\varepsilon}: I(\varepsilon) \rightarrow I(\varepsilon)$ (see (2.1)) acts in coordinates as follows: for even $m$,

$$
S_{\varepsilon}(x)= \begin{cases}x+\tau^{m+1} & \text { if } x \in G_{0}^{m}(\varepsilon), \\ x+\tau^{m+3} & \text { if } x \in G E_{0}^{m}(\varepsilon), \\ x-\tau^{m+2} & \text { if } x \in E_{0}^{m}(\varepsilon)\end{cases}
$$

and for odd $m$,

$$
S_{\varepsilon}(x)= \begin{cases}x+\tau^{m+2} & \text { if } x \in G_{0}^{m}(\varepsilon), \\ x-\tau^{m+3} & \text { if } x \in G E_{0}^{m}(\varepsilon), \\ x-\tau^{m+1} & \text { if } x \in E_{0}^{m}(\varepsilon) .\end{cases}
$$

Using the formula $\tau^{n}=(-1)^{n}\left(F_{n-1}-F_{n} \cdot \tau\right)$ for $n \geq 1$, we obtain the congruence $\tau^{n} \equiv(-1)^{n-1} F_{n} \cdot \tau \bmod 1$ for $n \geq 1$. Then the rearrangement of the intervals (2.9), (2.10) can be represented as multiple shifts: for even $m$,

$$
S_{\varepsilon}(x) \equiv\left\{\begin{array}{lll}
x+F_{m+1} \cdot \tau & \bmod 1 & \text { if } x \in G_{0}^{m}(\varepsilon), \\
x+F_{m+3} \cdot \tau & \bmod 1 & \text { if } x \in G E_{0}^{m}(\varepsilon), \\
x+F_{m+2} \cdot \tau & \bmod 1 & \text { if } x \in E_{0}^{m}(\varepsilon) ;
\end{array}\right.
$$

and for odd $m$,

$$
S_{\varepsilon}(x) \equiv\left\{\begin{array}{lll}
x+F_{m+2} \cdot \tau & \bmod 1 & \text { if } x \in G_{0}^{m}(\varepsilon), \\
x+F_{m+3} \cdot \tau & \bmod 1 & \text { if } x \in G E_{0}^{m}(\varepsilon), \\
x+F_{m+1} \cdot \tau & \bmod 1 & \text { if } x \in E_{0}^{m}(\varepsilon) .
\end{array}\right.
$$

Formulas (2.9) and (2.10) imply the following isomorphism condition for two induced mappings:

$$
S_{\varepsilon} \sim S_{\eta} \Leftrightarrow \varepsilon \sim \eta \bmod E_{+},
$$

where the equivalence $\varepsilon \sim \eta \bmod E_{+}$means that $\varepsilon / \eta \in E_{+}$; here $E_{+}=\left\{\tau^{m} ; m \in \mathbb{Z}\right\}$ is the subgroup of positive units in the group $\mathcal{O}^{\times}$of units of the Fibonacci ring $\mathcal{O}$.

2.3. Partitions of the half-open interval $J$. Consider the shift

$$
J \stackrel{T}{\longrightarrow} J: \quad x \mapsto T(x)=x+1 \quad \bmod \widetilde{\tau}
$$

of the half-open interval $J=[-1, \tau)$ of length $\widetilde{\tau}=\tau+1$. Since

$$
\delta^{\prime}(N)=N-[(N+1) \tau] \cdot \widetilde{\tau} \equiv N \bmod \widetilde{\tau}
$$

we see that $T$ and $\delta^{\prime}(N)$ satisfy the relation

$$
\delta^{\prime}(N)=T^{N}(0) \quad \text { for all } N \in \mathbb{Z} .
$$

We take a half-open interval $\gamma=\left[\gamma^{-}, \gamma^{+}\right)$in $J$ and consider the induced mapping $T_{\gamma}=$ $\left.T\right|_{\gamma}$, which exists because 1 and $\tilde{\tau}$ are incommensurable.

By analogy with (2.2), we define $\widetilde{\varepsilon}_{m}=\widetilde{\tau} \cdot \varepsilon_{m}=\widetilde{\tau}-\tau^{m-1}$ for $m=0,1,2, \ldots$ Suppose that the length $|\gamma|=\gamma^{+}-\gamma^{-}$of $\gamma$ satisfies the condition

$$
\widetilde{\tau}-\widetilde{\varepsilon}_{m+1}<|\gamma| \leq \widetilde{\tau}-\widetilde{\varepsilon}_{m} .
$$

Observe that $\gamma \subset J$ by assumption. Therefore, there always exists a unique $m$ for which inequalities (2.15) are valid. We split $\gamma$ into three half-open intervals

$$
\gamma=\gamma_{G} \oplus \gamma_{G E} \oplus \gamma_{E}
$$


of lengths

$$
\left|\gamma_{G}\right|=\tau^{m+1}-\delta, \quad\left|\gamma_{G E}\right|=\delta, \quad\left|\gamma_{E}\right|=\tau^{m}-\delta
$$

for even $m$, and

$$
\left|\gamma_{G}\right|=\tau^{m}-\delta, \quad\left|\gamma_{G E}\right|=\delta, \quad\left|\gamma_{E}\right|=\tau^{m+1}-\delta
$$

for odd $m$, where the continuous parameter

$$
\delta=\left(\widetilde{\tau}-\widetilde{\varepsilon}_{m}\right)-|\gamma|
$$

varies in the interval $0 \leq \delta<\tau^{m+1}$.

Relations (2.9), (2.10) and (2.11), (2.12) imply the following statement.

Theorem 2.1. Let $\gamma$ be a half-open interval in $J=[-1, \tau)$, and let $m=0,1,2, \ldots$ satisfy condition (2.15).

1. Then the induced mapping $T_{\gamma}=\left.T\right|_{\gamma}$ rearranges the half-open intervals in the partition (2.16) noncyclically,

$$
T_{\gamma}\left(\gamma_{G} \oplus \gamma_{G E} \oplus \gamma_{E}\right)=\gamma_{E} \oplus \gamma_{G E} \oplus \gamma_{G} .
$$

If $\delta=0$, then $T_{\gamma}$ degenerates into the rearrangement of two intervals; i.e., $T_{\gamma}$ is isomorphic to a rotation of the unit circle. In coordinates, the rearrangement (2.20) has the form

$$
T_{\gamma}\left(t^{\prime}\right)=t^{\prime}+\Delta_{\gamma}^{\prime}\left(t^{\prime}\right)
$$

where $\Delta_{\gamma}^{\prime}\left(t^{\prime}\right)=\tau^{m}, \tau^{m+2},-\tau^{m+1}$ for $t^{\prime} \in \gamma_{G}, \gamma_{G E}, \gamma_{E}$, respectively, if $m$ is even, and $\Delta_{\gamma}^{\prime}\left(t^{\prime}\right)=\tau^{m+1},-\tau^{m+2},-\tau^{m}$ for $t^{\prime} \in \gamma_{G}, \gamma_{G E}, \gamma_{E}$, respectively, if $m$ is odd.

2. Let $N$ belong to the set $N_{\gamma}$ of all $M \in \mathbb{Z}$ such that $\delta^{\prime}(M) \in \gamma$. Then formula (2.21) takes the form

$$
T_{\gamma} \delta^{\prime}(N)=\delta^{\prime}\left(N+\Delta_{\gamma}(N)\right)
$$

where $\Delta_{\gamma}(N)=F_{m+1}, F_{m+3}, F_{m+2}$ for $N \in N_{G}, N_{G E}, N_{E}$, respectively, if $m$ is even, and $\Delta_{\gamma}(N)=F_{m+2}, F_{m+3}, F_{m+1}$ for $N \in N_{G}, N_{G E}, N_{E}$, respectively, if $m$ is odd. The set $N_{\gamma}$ is split in accordance with the partition (2.16) of $\gamma$,

$$
N_{\gamma}=N_{G} \cup N_{G E} \cup N_{E},
$$

i.e., $N_{X}=\left\{N \in \mathbb{Z} ; \delta^{\prime}(N) \in \gamma_{X}\right\}$.

Two mappings $T_{\gamma_{1}}$ and $T_{\gamma_{2}}$ are similar, $T_{\gamma_{1}} \sim T_{\gamma_{2}}$, if they coincide after bringing the half-open intervals $\gamma_{1}$ and $\gamma_{2}$ to one and the same scale. Theorem 2.1 shows that the following statement is true.

Corollary 2.1. We have

$$
T_{\gamma_{1}} \sim T_{\gamma_{2}}^{ \pm 1} \Leftrightarrow\left|\gamma_{1}\right| \sim\left|\gamma_{2}\right| \bmod E_{+},
$$

where the sign in $T_{\gamma_{2}}^{ \pm 1}$ depends on whether the congruence $m_{1} \equiv m_{2} \bmod 2$ is fulfilled.

Any half-open interval $\gamma$ in $J$ gives rise to a one-dimensional quasilattice:

$$
\mathcal{L}^{1}=\mathcal{L}^{1}(\gamma)=\left\{t ; t^{\prime} \in \Gamma\right\} \subseteq \mathcal{F}^{1},
$$

where $\Gamma=\gamma \cap \mathcal{O}$. The definitions imply that the following diagram is commutative:

$$
\begin{array}{llll}
\Gamma & \stackrel{e m}{\hookrightarrow} & \mathcal{J} \\
\downarrow^{\prime} & & \downarrow^{\prime} \\
\mathcal{L}^{1} & \stackrel{e m}{\hookrightarrow} & \mathcal{F}^{1},
\end{array}
$$

where $\mathcal{J}=J \cap \mathcal{O}, \mathcal{F}^{1}=\mathcal{F}$, em are embeddings of sets, and ' denotes conjugation in the quadratic field $F$. 
We say that two one-dimensional quasilattices $\mathcal{L}^{1}\left(\gamma_{1}\right)$ and $\mathcal{L}^{1}\left(\gamma_{2}\right)$ in the square quasilattice $\mathcal{F}^{2}$ are similar, $\mathcal{L}^{1}\left(\gamma_{1}\right) \stackrel{\mathcal{s}}{\sim} \mathcal{L}^{1}\left(\gamma_{2}\right)$, if there is a similarity transformation of the line $\mathbb{R}^{1}$ that takes one quasilattice to the other.

Corollary 2.2. We have

$$
\mathcal{L}^{1}\left(\gamma_{1}\right) \stackrel{s}{\sim} \mathcal{L}^{1}\left(\gamma_{2}\right) \Leftrightarrow\left|\gamma_{1}\right| \sim\left|\gamma_{2}\right| \bmod E_{+} .
$$

This follows from Corollary 2.1 and diagram (2.26).

\section{$\S 3$. Homogeneous and nonhomogeneous Quasilattices $\mathcal{L}$}

3.1. Homogeneous and nonhomogeneous lines. We define a line $L$ over the Fibonacci ring $\mathcal{O}=\mathbb{Z}[\tau]$ by the equation

$$
a_{1} x_{1}+a_{2} x_{2}=0
$$

with $a_{i}$ and $x_{i}$ in $\mathcal{O}$, where $\left(a_{1}, a_{2}\right) \neq(0,0)$. Let $\beta=\left(\beta_{1}, \beta_{2}\right)$ be a primitive integral solution of equation (3.1), i.e., a nonzero solution with g.c.d. $\left(\beta_{1}, \beta_{2}\right)=1$. Such a solution always exists since $\mathcal{O}$ is factorial. Then $L$ is a module over $\mathcal{O}$ and $\beta$ is its generator,

$$
L=\mathcal{O}[\beta] .
$$

Indeed, let $x=\left(x_{1}, x_{2}\right) \neq(0,0)$ be an arbitrary solution of (3.1). Then $\beta_{1} x_{2}=\beta_{2} x_{1}$. Since g.c.d. $\left(\beta_{1}, \beta_{2}\right)=1$ by assumption, we have $x_{i}=k \beta_{i}, i=1,2$, for some $k \in \mathcal{O}$, i.e., $x=k \beta$.

In the case of a nonhomogeneous equation, we have

$$
a_{1} x_{1}+a_{2} x_{2}=c,
$$

where $c \in \mathcal{O}$, and the line $L$ can be represented in the form

$$
L=\mathcal{O}[\beta]+v,
$$

where $v=\left(v_{1}, v_{2}\right)$ is a fixed integral solution of (3.3), and $\beta$ is again a primitive solution of (3.1). If $c=0$, then we take $v=(0,0)$. Conversely, every pair $\beta, v$ of vectors in $\mathcal{O}^{2}$, where $\beta$ is a primitive vector, gives a line

$$
L=L(\beta, v)
$$

defined by an equation of the form (3.3).

3.2. One-dimensional quasilattices. Every line $L=L(\beta, v)$ gives rise to the subset

$$
\mathcal{L}=L \cap \mathcal{F}^{2}
$$

of the two-dimensional Fibonacci quasilattice $\mathcal{F}^{2}$. If $\mathcal{L}$ contains at least two points, then it contains infinitely many points (see Lemma 3.1 ).

In this case, the set $\mathcal{L}$ as in (3.6) is called a one-dimensional quasilattice in $\mathcal{F}^{2}$. In the sequel, we consider only lines $L$ with this property. We note that the quasilattice $\mathcal{L}$ determines the line $L$ uniquely.

The definition of the Fibonacci quasilattice $\mathcal{F}^{2}$ shows that, apart from (3.6), yet another representation for $\mathcal{L}$ is possible:

$$
\mathcal{L}=\left(L^{\prime} \cap J^{2}\right)^{\prime} .
$$

We say that a quasilattice $\mathcal{L}_{0}$ is homogeneous if the line $L_{0}$ passes through the origin; otherwise $\mathcal{L}$ is nonhomogeneous. With every nonhomogeneous quasilattice $\mathcal{L}$ we can associate a homogeneous quasilattice $\mathcal{L}_{0}$ generated by the line $L_{0}$ that is parallel to $L$ and passes through the origin. 
Lemma 3.1. Let $L(\beta, v)$ be a line with generating vector $\beta$ of length

$$
|\beta| \geq \widetilde{\tau}^{2} .
$$

Then there is an interval $\gamma$ in $J$ for which we have the following bijection:

$$
\Gamma \stackrel{\sigma}{\sim} \mathcal{L}: \quad t^{\prime} \mapsto x=\sigma\left(t^{\prime}\right)=t \cdot \beta+v
$$

where $\Gamma=\gamma \cap \mathcal{O}$ is a parameter set for the quasilattice $\mathcal{L}$.

Proof. Since the generating vector $\beta$ can be replaced by an arbitrary vector of the form $\tau^{m} \beta, m \in \mathbb{Z}$, we may assume that condition (3.8) is fulfilled. Let $\gamma$ be a maximal interval in $J$ such that $\sigma^{\prime}\left(t^{\prime}\right)=t^{\prime} \cdot \beta^{\prime}+v^{\prime} \in J$ for every $t^{\prime} \in \Gamma$. Let

$$
\mathcal{L}^{\prime}=L^{\prime} \cap J^{2} \subset J^{2}
$$

be the image of the quasilattice $\mathcal{L}$ under conjugation. Then (3.10) and (3.4) imply the relation $\mathcal{L}^{\prime}=\sigma^{\prime}(\Gamma)$, which is equivalent to the fact that the mapping

$$
\Gamma \stackrel{\sigma^{\prime}}{\sim} \mathcal{L}^{\prime}: t^{\prime} \mapsto x^{\prime}=\sigma^{\prime}\left(t^{\prime}\right)
$$

is bijective, and the lemma follows.

The bijection (3.9) gives a parametrization of the quasilattice $\mathcal{L} \subset \mathcal{F}^{2}$ by the points of the set $\Gamma$. Thus, the quasilattice

$$
\mathcal{L}=\mathcal{L}(\beta, v, \gamma)
$$

is uniquely determined by the vectors $\beta, v \in \mathcal{O}^{2}$ as in (3.5) such that $\beta$ is primitive and the conjugate line $L^{\prime}=\mathcal{O}\left[\beta^{\prime}\right]+v^{\prime}$ has a nonempty intersection with $J^{2}$. If, in addition, we assume that the length of $\beta$ satisfies (3.8), then in $J$ there is an interval $\gamma$ giving the parameter set $\Gamma=\gamma \cap \mathcal{O}$ for the quasilattice $\mathcal{L}=L \cap \mathcal{F}^{2}$.

3.3. The parameter set. We explicitly describe the intervals $\gamma_{0}$ for the case of homogeneous quasilattices $\mathcal{L}_{0}$ of the form (3.11) with $v=0$. For this, we must split the set of all vectors $\beta^{\prime}=\left(\beta_{1}^{\prime}, \beta_{2}^{\prime}\right)$ into three regions $S e c_{i}$ defined by the inequalities

$$
-\tau \leq \frac{\beta_{2}^{\prime}}{\beta_{1}^{\prime}} \leq 1, \quad-\tau \leq \frac{\beta_{1}^{\prime}}{\beta_{2}^{\prime}} \leq 1, \quad-\widetilde{\tau} \leq \frac{\beta_{2}^{\prime}}{\beta_{1}^{\prime}} \leq-\tau
$$

for $i=1,2,3$, respectively. Each region $S e c_{i}$ is the union of two centrally symmetric sectors. A direct calculation shows that, for homogeneous quasilattices $\mathcal{L}_{0}=\mathcal{L}_{0}\left(\beta, 0, \gamma_{0}\right)$ and vectors $\beta$ in $S e c_{i}$, the intervals $\gamma_{0}$ have the following form:

for $i=1$,

$$
\gamma_{0}=\left[\frac{-1}{\beta_{1}^{\prime}}, \frac{\tau}{\beta_{1}^{\prime}}\right) \quad \text { if } \beta_{1}^{\prime}>0, \quad \gamma_{0}=\left[\frac{\tau}{\beta_{1}^{\prime}}, \frac{-1}{\beta_{1}^{\prime}}\right) \quad \text { if } \beta_{1}^{\prime}<0
$$

for $i=2$,

$$
\gamma_{0}=\left[\frac{-1}{\beta_{2}^{\prime}}, \frac{\tau}{\beta_{2}^{\prime}}\right) \quad \text { if } \beta_{2}^{\prime}>0, \quad \gamma_{0}=\left[\frac{\tau}{\beta_{2}^{\prime}}, \frac{-1}{\beta_{2}^{\prime}}\right) \quad \text { if } \beta_{2}^{\prime}<0
$$

for $i=3$,

$$
\gamma_{0}=\left(\frac{\tau}{\beta_{2}^{\prime}}, \frac{\tau}{\beta_{1}^{\prime}}\right) \quad \text { if } \beta_{1}^{\prime}>0, \quad \gamma_{0}=\left(\frac{\tau}{\beta_{1}^{\prime}}, \frac{\tau}{\beta_{2}^{\prime}}\right) \quad \text { if } \beta_{1}^{\prime}<0 .
$$

By (3.13), the intervals $\gamma_{0}$ have the following lengths:

$$
\left|\gamma_{0}\right|=\frac{\widetilde{\tau}}{\left|\beta_{1}^{\prime}\right|} \quad \text { for } i=1, \quad\left|\gamma_{0}\right|=\frac{\widetilde{\tau}}{\left|\beta_{2}^{\prime}\right|} \quad \text { for } i=2, \quad\left|\gamma_{0}\right|=\tau\left|\frac{1}{\beta_{1}^{\prime}}-\frac{1}{\beta_{2}^{\prime}}\right| \quad \text { for } i=3
$$




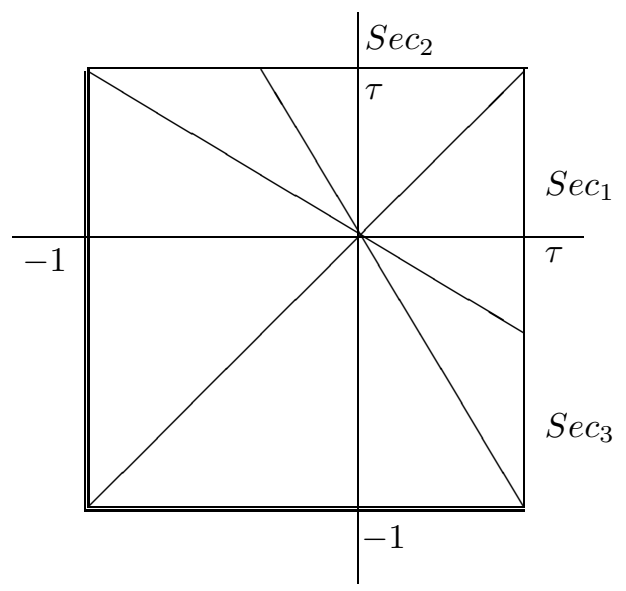

Figure 3.1. The interior space $J^{2}$

If $\mathcal{L}=\mathcal{L}(\beta, v, \gamma)$ is a nonhomogeneous quasilattice, then the length of the interval $\gamma$ can be found from the equation

$$
\frac{|\gamma|}{\left|\gamma_{0}\right|}=\frac{\left|\overline{\mathcal{L}^{\prime}}\right|}{\left|\overline{\mathcal{L}_{0}^{\prime}}\right|}
$$

where the intervals $\overline{\mathcal{L}^{\prime}}, \overline{\mathcal{L}_{0}^{\prime}} \subset \overline{J^{2}}$ are the closures of $\mathcal{L}^{\prime}$ and $\mathcal{L}_{0}^{\prime}$, respectively.

Since the lines $L^{\prime}$ and $L_{0}^{\prime}$ are parallel, we can often use shift by the vector $v^{\prime}$ to embed one of the sets $\mathcal{L}^{\prime}$ or $\mathcal{L}_{0}^{\prime}$ in the other. Therefore, there exists an embedding of the nonhomogeneous quasilattice $\mathcal{L}$ in the corresponding homogeneous quasilattice $\mathcal{L}_{0}$ (see Subsection 3.2), or vice versa. If the conjugate vector $\beta^{\prime}$ belongs to the region $\mathrm{Sec}_{3}$ (see Figure 3.1), then one of the quasilattices $\mathcal{L}$ or $\mathcal{L}_{0}$ can always be embedded in the other. If $\beta^{\prime}$ belongs to $S e c_{1}$ or to $S e c_{2}$, then $\mathcal{L}$ can be embedded in $\mathcal{L}_{0}$. Exceptions are possible only if $\left|\overline{\mathcal{L}^{\prime}}\right|=\left|\overline{\mathcal{L}_{0}^{\prime}}\right|$ and the line $L^{\prime}$ intersects, respectively, the axis $O Y$ or the axis $O X$ in a nonintegral point of the field $F$. In Figure 1.1 the nonhomogeneous quasilattice $\mathcal{L}_{2}$ embeds in the homogeneous quasilattice $\mathcal{L}_{2,0}$, via shifts by the vectors $v_{1}=(\delta(-2), 0)$ and $v_{2}=(0, \delta(-2))$. We have

$$
\mathcal{L}_{2,0}=\left(\mathcal{L}_{2}+v_{1}\right) \cup\left(\mathcal{L}_{2}+v_{2}\right) .
$$

\section{§4. LOCAL VECTORS AND LOCAL NUMBERS IN A QUASILATtice $\mathcal{L}$}

Let $\mathcal{L}=\mathcal{L}(\beta, v, \gamma)$ be the quasilattice (3.11). The length $|\gamma|$ of the corresponding interval $\gamma$ satisfies

$$
\tau^{m}<|\gamma| \leq \tau^{m-1}
$$

for some level $m=0,1,2, \ldots$. Suppose that the parameter set $\Gamma=\gamma \cap \mathcal{O}$ is split in accordance with the partition (2.16) of the interval $\gamma$,

$$
\Gamma=\Gamma_{G} \cap \Gamma_{G E} \cap \Gamma_{E} .
$$

Theorem 4.1. 1. Let $y$ be a point of the quasilattice $\mathcal{L}=\mathcal{L}(\beta, v, \gamma)$ neighboring to the point $x=\sigma\left(t^{\prime}\right)$ (see (3.9)) in the direction of the vector $\beta$. If $T_{\gamma}\left(t^{\prime}\right) \in \Gamma$, where $T_{\gamma}$ is the induced mapping described in Theorem 2.1, then

$$
y=x+\Delta_{\mathcal{L}}\left(t^{\prime}\right)
$$


where $\Delta_{\mathcal{L}}\left(t^{\prime}\right)$ is the local vector of the point $x=\sigma\left(t^{\prime}\right)$, calculated by the formulas

$$
\begin{array}{lll}
\Delta_{\mathcal{L}}\left(t^{\prime}\right)=\widetilde{\tau}^{m} \beta, \widetilde{\tau}^{m+2} \beta, \widetilde{\tau}^{m+1} \beta & \text { for } t^{\prime} \in \Gamma_{G}, \Gamma_{G E}, \Gamma_{E} \quad \text { if } m \text { is even, } \\
\Delta_{\mathcal{L}}\left(t^{\prime}\right)=\widetilde{\tau}^{m+1} \beta, \widetilde{\tau}^{m+2} \beta, \widetilde{\tau}^{m} \beta & \text { for } t^{\prime} \in \Gamma_{G}, \Gamma_{G E}, \Gamma_{E} \quad \text { if } m \text { is odd. }
\end{array}
$$

2. The point $x=\sigma\left(t^{\prime}\right)=\left(\delta\left(N_{1}\right), \delta\left(N_{2}\right)\right)$ has the following numbers $N_{1}, N_{2} \in \mathbb{Z}$ :

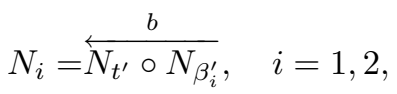

where $\circ$ denotes Knuth's circular multiplication (5.18), $t^{\prime}=\delta^{\prime}\left(N_{t^{\prime}}\right)$, and the coordinates of the vector $\beta^{\prime}=\left(\beta_{1}^{\prime}, \beta_{2}^{\prime}\right)$ have the form

$$
\beta_{i}^{\prime}=\widetilde{\tau}^{b} \cdot \delta^{\prime}\left(N_{\beta_{i}^{\prime}}\right), \quad i=1,2 .
$$

Here $b=0,1,2, \ldots$ is the power ensuring (4.5) with some $N_{\beta_{i}^{\prime}} \in \mathbb{Z}$. The numbers of the point $y$ neighboring to $x$ are calculated by the formula

$$
N_{i}+\Delta_{\mathcal{L}}\left(N_{i}\right), \quad \text { where } \Delta_{\mathcal{L}}\left(N_{i}\right)=\overleftarrow{\left(N_{t^{\prime}}+\Delta_{\gamma}\left(N_{t^{\prime}}\right)\right) \circ N_{\beta_{i}^{\prime}}}-\overleftarrow{b}
$$

they are local numbers and $\Delta_{\gamma}\left(N_{t^{\prime}}\right)$ are the shifts (2.22).

Remark 4.1. Theorem 2.1 shows that the set of points $t^{\prime} \in \Gamma$ for which $T_{\gamma}\left(t^{\prime}\right) \notin \Gamma$ can contain at most two points $\gamma^{-}$and $\gamma^{+}$, which are the endpoints of the interval $\gamma=\left[\gamma^{-}, \gamma^{+}\right]=\bar{\Gamma}$. If $T_{\gamma}\left(t^{\prime}\right) \notin \Gamma$, then the point neighboring to $x=\sigma\left(t^{\prime}\right)$ is $y=\sigma\left(T_{\gamma}^{2}\left(t^{\prime}\right)\right)$; i.e., the expected point $y=\sigma\left(T_{\gamma}\left(t^{\prime}\right)\right)$ is skipped. This case corresponds to the quasilattice $\mathcal{L}_{1}$ in Figure 1.1. For all other quasilattices, we have $T_{\gamma}\left(t^{\prime}\right) \in \Gamma$.

Proof. Consider the commutative diagram (see (2.26) $)$

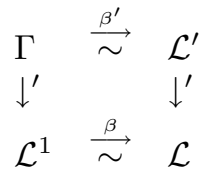

in which, by the parametrization (3.9), all mappings are bijections: the upper arrow is the mapping $t^{\prime} \mapsto t^{\prime} \cdot \beta^{\prime}$, and the lower arrow is the affine mapping $t \mapsto t \cdot \beta$. Since an affine mapping preserves the order of the points, we see that (2.21) implies the first statement of the theorem.

The number $b$ mentioned in (4.5) exists indeed, because it can be found from the condition that $\beta_{i}^{\prime} / \widetilde{\tau}^{b}$ is in the half-open interval $J=[-1, \tau)$. By assumption, $t^{\prime} \beta_{1}^{\prime}=$ $\delta^{\prime}\left(N_{t^{\prime}}\right) \beta_{1}^{\prime}$ belongs to $J$. Therefore,

$$
\delta^{\prime}\left(N_{t^{\prime}}\right) \beta_{i}^{\prime}=\widetilde{\tau}^{b} \delta^{\prime}\left(N_{t^{\prime}}\right) \delta^{\prime}\left(N_{\beta_{i}^{\prime}}\right)=\widetilde{\tau}^{b} \delta^{\prime}\left(N_{t^{\prime}} \circ N_{\beta_{i}^{\prime}}\right)=\delta^{\prime}\left(\overleftarrow{N_{t^{\prime}} \circ N_{\beta_{i}^{\prime}}}\right)
$$

This proves relation (4.5); combining this with (2.22), we get formula (4.7).

\section{§5. Distribution and Density of points in a QUasilattice $\mathcal{L}$. Applications to Diophantine EQUations}

5.1. We split the set of points $x=\sigma\left(t^{\prime}\right)$ lying in a quasilattice $\mathcal{L}=\mathcal{L}(\beta, v, \gamma)$ into types $G, G E$, and $E$,

$$
\mathcal{L}=\mathcal{L}_{G} \cup \mathcal{L}_{G E} \cup \mathcal{L}_{E}
$$

in the following way: $x$ is of type $G, G E$, or $E$ if the parameter $t^{\prime}$ belongs to $\Gamma_{G}, \Gamma_{G E}$, or $\Gamma_{E}$, respectively (see (4.2)).

We assume that for the direction vector $\beta=\left(\beta_{1}, \beta_{2}\right)$ we have $\beta_{1} \neq 0$. Let $\operatorname{pr}_{1} x=x_{1}$ be the projection of $x=\left(x_{1}, x_{2}\right)$ to the axis $O X$. 
For each type of points $x=x_{Y}$, where $Y=G, G E$, or $E$, we define the frequency

$$
p_{Y}=\lim _{X \rightarrow \infty} \frac{\sharp\left(\operatorname{pr}_{1} \mathcal{L}_{Y} \cap\left[X_{1}, X_{1}+X\right]\right)}{\sharp\left(\operatorname{pr}_{1} \mathcal{L} \cap\left[X_{1}, X_{1}+X\right]\right)}
$$

of its occurrence in the quasilattice $\mathcal{L}$. For the quasilattice $\mathcal{L}=\mathcal{L}(\beta, v, \gamma)$, the level $m$ can be found from (4.1). If

$$
\tau^{m}<|\gamma|<\tau^{m-1},
$$

then we say that $\mathcal{L}$ is a nondegenerate quasilattice; if

$$
|\gamma|=\tau^{m-1},
$$

we say that the quasilattice is degenerate.

Theorem 5.1. 1. If $\mathcal{L}=\mathcal{L}(\beta, v, \Gamma)$ is a one-dimensional quasilattice in $\mathcal{F}^{2}$ for which the length of the interval $\gamma$ satisfies inequalities (5.2), then the frequencies $p_{Y}$ of its points $x_{Y} \in \mathcal{L}$ can be calculated by the formulas

$$
p_{G}=1-\frac{\tau^{m}}{|\gamma|}, \quad p_{G E}=\frac{\tau^{m-1}}{|\gamma|}-1, \quad p_{E}=1-\frac{\tau^{m+1}}{|\gamma|}
$$

if the level $m$ is even, and by

$$
p_{G}=1-\frac{\tau^{m+1}}{|\gamma|}, \quad p_{G E}=\frac{\tau^{m-1}}{|\gamma|}-1, \quad p_{E}=1-\frac{\tau^{m}}{|\gamma|}
$$

if $m$ is odd.

2. If the quasilattice $\mathcal{L}$ is degenerate, i.e., if (5.3) is true, then the frequencies are calculated by the formulas

$$
p_{G}=\tau^{2}, \quad p_{G E}=0, \quad p_{E}=\tau
$$

if $m$ is even, and by the formulas

$$
p_{G}=\tau, \quad p_{G E}=0, \quad p_{E}=\tau^{2}
$$

if $m$ is odd.

3. Let $n_{\mathcal{L}}(X)$ be the number of points $x=\left(x_{1}, x_{2}\right)$ in $\mathcal{L}=\mathcal{L}(\beta, v, \Gamma)$ with $0 \leq x_{1} \leq X$, and let $\beta_{1} \neq 0$ for the direction vector $\beta=\left(\beta_{1}, \beta_{2}\right)$. Then

$$
n_{\mathcal{L}}(X)=k X+r_{\mathcal{L}}(X)
$$

with $k=\frac{|\gamma|}{c\left|\beta_{1}\right|}$, where $c=1+2 \tau$, and for the remainder term we have $r_{\mathcal{L}}(X)=o(X)$ as $X \rightarrow+\infty$.

Proof. 1-2. If the length of $\gamma$ satisfies (5.2) and $m$ is even, then, by (2.19), we have $\delta=\left(\widetilde{\tau}-\widetilde{\varepsilon}_{m}\right)-|\gamma|=\tau^{m-1}-|\gamma|$, and (2.17) implies

$$
\left|\gamma_{G}\right|=|\gamma|-\tau^{m}, \quad\left|\gamma_{G E}\right|=\tau^{m-1}-|\gamma|, \quad\left|\gamma_{E}\right|=|\gamma|-\tau^{m+1} .
$$

Combining this with the definition (3.9) of the bijection $\sigma$, we obtain formulas (5.4). Formulas (5.5)-(5.7) are proved similarly.

3. The relation $X=\delta\left(N_{X}\right)$ yields

$$
N_{X}=\frac{X}{c_{1}}+c_{2}(X), \quad \text { where } c_{1}=1+\tau^{2}, \quad \frac{-\tau^{3}}{1+\tau^{2}}<c_{2}(X)<\widetilde{\tau} .
$$

The first coordinates of the points $x=\sigma\left(t^{\prime}\right)$ with parameter $t^{\prime}=\delta^{\prime}\left(N_{t^{\prime}}\right)$ satisfy the inequality $x_{1}=t \cdot \beta_{1} \leq X$. Therefore, $\delta\left(N_{t^{\prime}}\right) \leq X / \beta_{1}$, and, by (5.9),

$$
N_{t^{\prime}} \leq \frac{X}{c_{1} \beta_{1}}+c_{3}\left(\frac{X}{\beta_{1}}\right)
$$


TABLE 5.1

\begin{tabular}{|c|c|c|c|c|c|c|}
\hline $\mathcal{L}$ & $L$ & $m$ & $k$ & $p_{G}$ & $p_{G E}$ & $p_{E}$ \\
\hline $\mathcal{F}$ & $x_{2}=0$ & 0 & $\frac{1+\tau}{1+2 \tau} \approx 0.72$ & $\tau^{2} \approx 0.38$ & 0 & $\tau \approx 0.62$ \\
\hline $\mathcal{L}_{1}$ & $\tau x_{1}-x_{2}=0$ & 1 & $\frac{1}{1+2 \tau} \approx 0.45$ & $\tau \approx 0.62$ & 0 & $\tau^{2} \approx 0.38$ \\
\hline $\mathcal{L}_{2,0}$ & $x_{1}+x_{2}=0$ & 0 & $\frac{2 \tau}{1+2 \tau} \approx 0.55$ & $\frac{\tau^{2}}{2} \approx 0.19$ & $\frac{\tau}{2} \approx 0.31$ & $\frac{1}{2}=0.5$ \\
\hline $\mathcal{L}_{2}$ & $x_{1}+x_{2}=2+\tau$ & 1 & $\frac{\tau+\tau^{3}}{1+2 \tau} \approx 0.39$ & $\frac{2 \tau^{2}}{1+\tau^{2}} \approx 0.55$ & $\frac{\tau^{3}}{1+\tau^{2}} \approx 0.17$ & $\frac{\tau^{2}}{1+\tau^{2}} \approx 0.28$ \\
\hline $\mathcal{L}_{3}$ & $(2+\tau) x_{1}-x_{2}=0$ & 0 & $\frac{1+\tau}{1+2 \tau} \approx 0.72$ & $\tau^{2} \approx 0.38$ & 0 & $\tau \approx 0.62$ \\
\hline
\end{tabular}

The fraction of points $x=\sigma\left(t^{\prime}\right)$ in $\mathcal{L}$ with numbers $N_{t^{\prime}} \geq 0$ satisfying (5.10) is $\frac{|\gamma|}{|J|}=\frac{|\gamma|}{\widetilde{\tau}}$. Therefore, (5.10) implies (5.8).

We apply (5.8) to the quasilattices $\mathcal{L}$ presented in Figure 1.1. For each quasilattice $\mathcal{L}=L \cap \mathcal{F}^{2}$ of this sort, Table 5.1 contains an equation of the corresponding line $L$, the level $m$ (see (5.2), (5.3)), the corresponding coefficient $k$ (see (5.8)), and the frequencies $p_{G}, p_{G E}$, and $p_{E}($ see (5.4)-(5.7) $)$.

5.2. Systems of Diophantine equations. As a special case of Theorem 5.1, we obtain the following statement.

Proposition 5.1. Let

$$
a_{1} N_{1}+a_{2} N_{2}=c_{1}, \quad a_{1}\left[N_{1} \tau\right]+a_{2}\left[N_{2} \tau\right]=c_{2}
$$

be a system of Diophantine equations in which the coefficients $a_{i}, c_{i}$ and the variables $N_{i}$ belong to the ring $\mathbb{Z}$ of rational integers. Suppose that the coefficients satisfy the following conditions:

$$
\begin{aligned}
& \text { g.c.d. }\left(a_{1}, a_{2}\right)=1, \quad a_{2} \geq-a_{1} \geq 3, \\
& c^{\prime}>0, \quad m<c^{\prime}<M, \quad-1<\frac{c^{\prime}-a_{2} \tau}{a_{1}}<\tau,
\end{aligned}
$$

where $c^{\prime}=c_{1}-c_{2} \widetilde{\tau}-a_{1}-a_{2}$ and $m$ is the minimal and $M$ the maximal element in $\left\{a_{1} x_{1}+a_{2} x_{2} ; x_{1}=\tau,-1, x_{2}=\tau,-1\right\}$. Then system (5.11) has a solution, and the number $n(X)$ of its solutions $\left(N_{1}, N_{2}\right)$ with $X_{1} \leq N_{1} \leq X_{1}+X$, where $X_{1}$ is fixed and $X \rightarrow+\infty$, satisfies the asymptotic relation

$$
n(X)=k X+o(X), \quad \text { where } k=\frac{\left|a_{2}-c_{1} \tau+c_{2}\right|}{\left|a_{1}\right| a_{2}^{2}} .
$$

Proof. Consider the line $L$ given by the equation

$$
a_{1} x_{1}+a_{2} x_{2}=\widetilde{c},
$$

where $\widetilde{c}=c_{1}-a_{1}-a_{2}+c_{2} \tau$ and $a_{i}, c_{i} \in \mathbb{Z}$. Then the quasilattice $\mathcal{L}$ consists of all points $\left(x_{1}, x_{2}\right)$ satisfying (5.15) and having the form $\left(\delta\left(N_{1}\right), \delta\left(N_{2}\right)\right)$, where $\delta(N)=$ $N+[(N+1) \tau] \tau, N_{i} \in \mathbb{Z}$. Substituting such $\left(x_{1}, x_{2}\right)$ in (5.15), we obtain a system equivalent to (5.11).

Since $a_{1}$ and $a_{2}$ are relatively prime, we see that the conjugate line $L^{\prime}$ contains a point of $\mathcal{O}^{2}$, and conditions (5.13) imply that $L^{\prime}$ has a nonempty intersection with the square $J^{2}$. Therefore, equation (5.15) is solvable, and so is system (5.11). 
By conditions (5.13), the direction vector $\beta^{\prime}=\beta=\left(a_{2},-a_{1}\right)$ belongs to $S e c_{1}$. For the homogeneous quasilattice $\mathcal{L}_{0}$, we have $\gamma_{0}=\left[\frac{-1}{a_{2}}, \frac{\tau}{a_{2}}\right)$ by formula (3.13) (the case where $i=1$ ). Therefore, $\frac{\left|\gamma_{0}\right|}{|J|}=\frac{1}{a_{2}}$. Using relations (5.13) once again, we obtain

$$
\frac{|\gamma|}{\left|\gamma_{0}\right|}=\frac{\frac{\widetilde{c}^{\prime}-a_{2} \tau}{a_{1}}+1}{\widetilde{\tau}}
$$

Using this formula, relation $k=\frac{\left|\gamma_{0}\right|}{|J|} \cdot \frac{|\gamma|}{\left|\gamma_{0}\right|} \cdot \frac{1}{a_{2}}$, and (5.8), we arrive at (5.14).

5.3. Linear equations over the Fibonacci o-ring. The numbers $F_{n}$, with initial conditions $F_{1}=1$ and $F_{2}=2$, form the Fibonacci scale of notation, in which every positive integer $N$ can be represented uniquely as a finite sum of the form

$$
N=\varepsilon_{1} F_{1}+\varepsilon_{2} F_{2}+\cdots+\varepsilon_{n} F_{n}+\cdots=\sum_{n \geq 1} \varepsilon_{n}(N) F_{n},
$$

where the coefficients $\varepsilon_{n}=0,1$ have the cancelation property $\varepsilon_{n} \varepsilon_{n+1}=0$ for all $n \geq 1$. In the Fibonacci scale of notation, the o-product (or Knuth's product [6]) $N_{1} \circ N_{2}$ is calculated by the formula

$$
N_{1} \circ N_{2}=\sum_{n \geq 1} \sum_{m \geq 1} \varepsilon_{n}\left(N_{1}\right) \varepsilon_{n}\left(N_{2}\right) F_{n+m},
$$

where $N_{1}, N_{2} \in \mathbb{N}$.

Remark. After this paper had been submitted for publication, the author was informed by $\mathrm{Yu}$. V. Matiyasevich that the circular multiplication (5.17) was introduced by him in the papers [8, 9] in 1968.

In [3], it was proved that the o-product can be written as

$$
N_{1} \circ N_{2}=N_{1} N_{2}+\left[\left(N_{1}+1\right) \tau\right]\left[\left(N_{2}+1\right) \tau\right]
$$

in terms of the function [*]; the same paper contains also the following quasi-invariance formulas:

$$
\begin{aligned}
\delta^{\prime}\left(N_{1}+N_{2}\right) & =\delta^{\prime}\left(N_{1}\right)+\delta^{\prime}\left(N_{2}\right) & & \text { if } \delta^{\prime}\left(N_{1}\right)+\delta^{\prime}\left(N_{2}\right) \in J \\
\delta^{\prime}\left(N_{1} \circ N_{2}\right) & =\delta^{\prime}\left(N_{1}\right) \cdot \delta^{\prime}\left(N_{2}\right) & & \text { if } \delta^{\prime}\left(N_{1}\right) \cdot \delta^{\prime}\left(N_{2}\right) \in J
\end{aligned}
$$

We denote by $\stackrel{\circ}{\mathbb{Z}}=\mathbb{Z}[+, \times, \circ]$ the ring $\mathbb{Z}$ of integers equipped with the extra operation (5.18) and call $\mathbb{Z}$ the Fibonacci o-ring. In Proposition 5.2, we consider yet another application of Theorem 5.1 to Diophantine equations over the o-ring $\stackrel{\circ}{\mathbb{Z}}$.

Proposition 5.2. Let

$$
A_{1} \circ X_{1}-A_{2} \circ X_{2}=C
$$

be an equation in which the coefficients $A_{i}$ and $C$ and the variables $X_{i}$ take values in $\mathbb{N}=\{1,2,3, \ldots\}$ and

$$
\left|\delta^{\prime}\left(A_{i}\right)\right| \leq \tau^{3}, \quad \text { g.c.d. }\left(n_{F / \mathbb{Q}}\left(\delta\left(A_{1}\right)\right), n_{F / \mathbb{Q}}\left(\delta\left(A_{2}\right)\right)\right)=1,
$$

where

$$
n_{F / \mathbb{Q}}(\delta(A))=A^{2}-A[(A+1) \tau]-[(A+1) \tau]^{2}
$$

is the norm of the element $\delta(A)=A+[(A+1) \tau] \tau$ in the quadratic extension $F / \mathbb{Q}$. We assume that either

$$
0<\frac{\delta^{\prime}\left(A_{2}\right)}{\delta^{\prime}\left(A_{1}\right)}<1, \quad \text { and then } m<\delta^{\prime}(C)<M,
$$


where $m$ is the minimal and $M$ the maximal number in the collection $\left\{\delta^{\prime}\left(A_{1}\right) \tau-\delta^{\prime}\left(A_{2}\right) \tau\right.$, $\left.-\delta^{\prime}\left(A_{1}\right)+\delta^{\prime}\left(A_{2}\right)\right\}$, or

$$
-\tau<\frac{\delta^{\prime}\left(A_{2}\right)}{\delta^{\prime}\left(A_{1}\right)}<0, \quad \text { and then } \quad m<\delta^{\prime}(C)<M,
$$

where $m$ is the minimum and $M$ is the maximum number in $\left\{\delta^{\prime}\left(A_{1}\right) \tau+\delta^{\prime}\left(A_{2}\right)\right.$, $\left.-\delta^{\prime}\left(A_{1}\right)-\delta^{\prime}\left(A_{2}\right) \tau\right\}$. Then, as $X \rightarrow+\infty$, the number $n(X)$ of solutions $\left(X_{1}, X_{2}\right)$ of equation (1.1) with $1 \leq X_{2} \leq X$ is calculated by the asymptotic formula

$$
n(X)=k X+o(X), \quad \text { where } k=\frac{1}{\left|n_{F / \mathbb{Q}}\left(\delta\left(A_{1}\right)\right)\right|} .
$$

Proof. We have $\delta^{\prime}\left(A_{i} \circ X_{i}\right)=\delta^{\prime}\left(A_{i}\right) \delta^{\prime}\left(X_{i}\right),\left|\delta^{\prime}\left(A_{i}\right) \delta^{\prime}\left(X_{i}\right)\right| \leq \tau^{3}$, and $\left|\delta^{\prime}\left(A_{1} \circ X_{1}\right)\right|+$ $\left|\delta^{\prime}\left(A_{2} \circ X_{2}\right)\right| \leq 2 \tau^{3}<\tau$; hence formulas (5.19) imply the relation

$$
\begin{gathered}
\delta^{\prime}\left(A_{1} \circ X_{1}-A_{2} \circ X_{2}\right)=\delta^{\prime}\left(A_{1} \circ X_{1}\right)-\delta^{\prime}\left(A_{2} \circ X_{2}\right) \\
=\delta^{\prime}\left(A_{1}\right) \delta^{\prime}\left(X_{1}\right)-\delta^{\prime}\left(A_{2}\right) \delta^{\prime}\left(X_{2}\right)=\delta^{\prime}(C) .
\end{gathered}
$$

We put $a_{i}^{\prime}=\delta^{\prime}\left(A_{i}\right), x_{i}^{\prime}=\delta^{\prime}\left(X_{i}\right)$, and $c^{\prime}=\delta^{\prime}(C)$. Then, by the second condition in (5.21), the conjugate line $L^{\prime}$ with equation $a_{1}^{\prime} x_{1}-a_{2}^{\prime} x_{2}=c^{\prime}$ has a point over the ring $\mathcal{O}$, and by (5.22) and (5.23), the intersection of $L^{\prime}$ and $J^{2}$ is an open interval. Applying Theorem 5.1 to the line $L$ with equation $a_{1} x_{1}-a_{2} x_{2}=c$, we obtain (5.24).

\section{§6. Classification of One-Dimensional Quasilattices}

6.1. Fine classification: similarity of quasilattices. We recall that two one-dimensional quasilattices $\mathcal{L}_{1}, \mathcal{L}_{2} \subset \mathcal{F}^{2}$ are said to be similar, $\mathcal{L}_{1} \stackrel{\mathcal{s}}{\sim} \mathcal{L}_{2}$, if there is a similarity of the plane $\mathbb{R}^{2}$ that takes one quasilattice to the other (cf. the definition in Subsection 2.3).

Theorem 6.1. Let $\mathcal{L}_{1}$ and $\mathcal{L}_{2}$ be nondegenerate quasilattices in $\mathcal{F}^{2}$. Then

$$
\mathcal{L}_{1} \stackrel{s}{\sim} \mathcal{L}_{2} \Leftrightarrow\left|\gamma_{1}\right| \sim\left|\gamma_{2}\right| \bmod E_{+} .
$$

Proof. We use formulas (5.4) and (5.5):

if $m$ is even and $\tau^{m}<|\gamma|<\tau^{m-1}$, then

$$
p_{G}=1-\frac{\tau^{m}}{|\gamma|}, \quad p_{G E}=\frac{\tau^{m-1}}{|\gamma|}-1, \quad p_{E}=1-\frac{\tau^{m+1}}{|\gamma|}
$$

if $m$ is odd and $\tau^{m}<|\gamma|<\tau^{m-1}$, then

$$
p_{G}=1-\frac{\tau^{m+1}}{|\gamma|}, \quad p_{G E}=\frac{\tau^{m-1}}{|\gamma|}-1, \quad p_{E}=1-\frac{\tau^{m}}{|\gamma|} .
$$

The similarity $\mathcal{L}_{1} \stackrel{s}{\sim} \mathcal{L}_{2}$ implies that the quasilattices have one and the same frequency distribution of points,

$$
p_{G, 1}=p_{G, 2}, \quad p_{G E, 1}=p_{G E, 2}, \quad p_{E, 1}=p_{E, 2} .
$$

Suppose the levels $m_{1}$ and $m_{2}$ are even. By formulas (6.2), we have $p_{G, 1}=1-\frac{\tau^{m_{1}}}{|\gamma|}$ and $p_{G, 2}=1-\frac{\tau^{m_{2}}}{|\gamma|}$. Therefore, the first equation in (6.4) implies $\left|\gamma_{1}\right|=\tau^{m_{1}-m_{2}}\left|\gamma_{2}\right|$, i.e., $\left|\gamma_{1}\right| \sim\left|\gamma_{2}\right|$. The remaining cases are analyzed similarly.

The converse follows from Corollary 2.2 and the commutative diagram (4.8). 


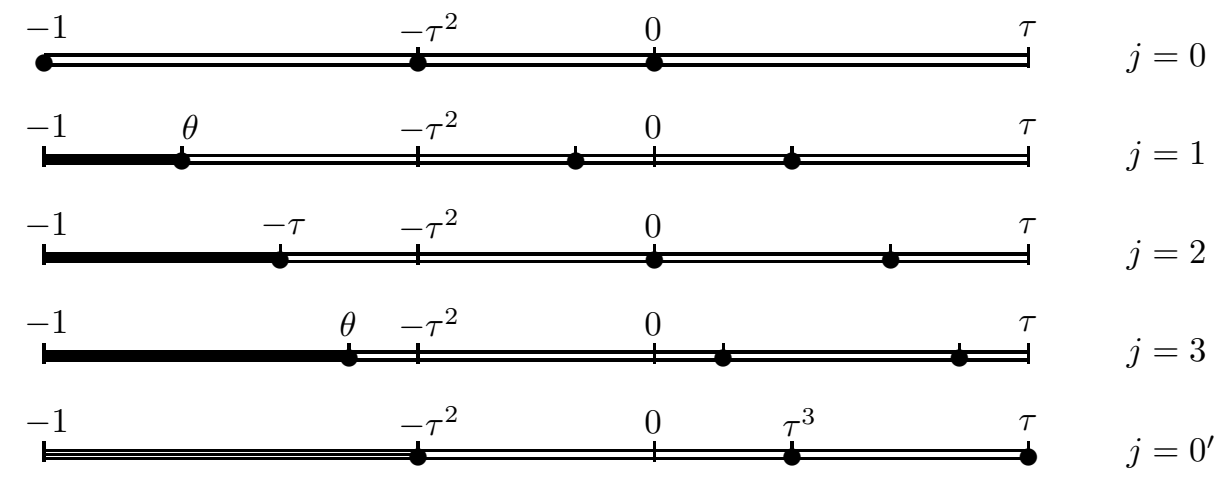

Figure 6.1. Symmetric Fibonacci partitions $P^{0}(|\gamma|)$

6.2. Rough classification: local equivalence of quasilattices. We assume that the half-open interval $\gamma$ has level $m$ determined by (4.1). We write

$$
\gamma=P^{m}(|\gamma|)+t_{0}^{\prime},
$$

where $P^{m}(|\gamma|)=[\theta, \tau)$ is the half-open interval attached to the endpoint of $J=[-1, \tau)$, and $\theta=\tau-|\gamma|$. Since $\gamma \subseteq J$ by assumption, we have $\theta \in[-1, \tau)$. If $m=0$, then, for the half-open interval $[\theta, \tau)$, we define the following partitions $P^{0}(|\gamma|)$ depending on the value of $\theta$ (see Figure 6.1): for $\theta \in[-1,-\tau$ ), we put

$$
P^{0}(|\gamma|)=P^{0}(1, \tau) \oplus P^{0}\left(\tau^{2}, \tau\right) \oplus P^{0}(-\tau, \tau) \oplus P^{0}\left(-\tau,-\tau^{2}\right) \oplus P^{0}(-\tau,-1),
$$

where

$$
\begin{gathered}
P^{0}(1, \tau)=\left[\theta,-\tau^{2}\right), \quad P^{0}\left(\tau^{2}, \tau\right)=\left[-\tau^{2},-\tau^{2}+\theta+1\right), \quad P^{0}(-\tau, \tau)=\left[-\tau^{2}+\theta+1,0\right), \\
P^{0}\left(-\tau,-\tau^{2}\right)=[0, \theta+1), \quad P^{0}(-\tau,-1)=[\theta+1, \tau) ;
\end{gathered}
$$

for $\theta \in\left[-\tau,-\tau^{2}\right)$, we put

$$
P^{0}(|\gamma|)=P^{0}(1, \tau) \oplus P^{0}\left(\tau^{2}, \tau\right) \oplus P^{0}\left(\tau^{2},-\tau^{2}\right) \oplus P^{0}\left(-\tau,-\tau^{2}\right) \oplus P^{0}(-\tau,-1),
$$

where

$$
\begin{gathered}
P^{0}(1, \tau)=\left[\theta,-\tau^{2}\right), \quad P^{0}\left(\tau^{2}, \tau\right)=\left[-\tau^{2}, 0\right), \quad P^{0}\left(\tau^{2},-\tau^{2}\right)=[0, \theta+\tau), \\
P^{0}\left(-\tau,-\tau^{2}\right)=[\theta+\tau, \theta+1), \quad P^{0}(-\tau,-1)=[\theta+1, \tau) .
\end{gathered}
$$

For an arbitrary level $m$, the partitions

$$
P^{m}\left(\left|\gamma_{m}\right|\right)=\tau^{m} \cdot\left(P^{0}\left(\widetilde{\tau} \cdot\left|\gamma_{0}\right|\right)-\tau\right)+\tau
$$

are obtained from the partitions (6.6), (6.7) of zero level by the similarity transformation with coefficient $\tau^{m}$ and center $\tau$. We denote by $P^{m}(|\gamma|)$ the interval $[\theta, \tau)$ itself and its partitions (6.8).

The partitions (6.8) yield rearrangements

$$
\mathcal{S}_{\theta}^{ \pm 1}: P^{m}(|\gamma|) \rightarrow P^{m}(|\gamma|)
$$

of the half-open intervals $P^{m}\left(t_{-}^{\prime}, t_{+}^{\prime}\right)$ in $P^{m}(|\gamma|)$ : we put

$$
\begin{aligned}
\mathcal{S}_{\theta}\left(t^{\prime}\right)=t^{\prime}+t_{+}^{\prime} & \text { if } t^{\prime} \in P^{m}\left(t_{-}^{\prime}, t_{+}^{\prime}\right), \\
\mathcal{S}_{\theta}^{-1}\left(t^{\prime}\right)=t^{\prime}+t_{-}^{\prime} & \text { if } t^{\prime} \in P^{m}\left(t_{-}^{\prime}, t_{+}^{\prime}\right) .
\end{aligned}
$$

Here, in $P^{m}\left(t_{-}^{\prime}, t_{+}^{\prime}\right)$, the shifts $t_{-}^{\prime}$ and $t_{+}^{\prime}$ are obtained from the corresponding shifts of level $m=0$ by multiplication by $\tau^{m}$. The partitions $P^{m}(|\gamma|)$ are obtained from the 
partitions $I(\varepsilon)$ with the parameter $\varepsilon=\tau(\theta+1)$ (see (2.7) ) by symmetrization. Moreover, we have an isomorphism

$$
\mathcal{S}_{\theta} \sim S_{\varepsilon}
$$

where $S_{\varepsilon}$ is the rearrangement (2.8) of intervals of the partition $I(\varepsilon)$.

If a half-open interval $\gamma$ has level $m=0$, then its length lies in the range $1<|\zeta| \leq \widetilde{\tau}$ (see (2.15)). We split $P^{0}=(1, \widetilde{\tau}]$ as follows:

$$
P^{0}=P_{3}^{0} \oplus P_{2}^{0} \oplus P_{1}^{0} \oplus P_{0}^{0},
$$

where $P_{3}^{0}=\left(1,1+\tau^{3}\right), P_{2}^{0}=\left\{1+\tau^{3}\right\}, P_{1}^{0}=\left(1+\tau^{3}, \widetilde{\tau}\right)$, and $P_{0}^{0}=\{\widetilde{\tau}\}$. We take $P^{0}$ as the basic half-open interval where $|\gamma|$ varies. Then $(0, \widetilde{\tau}]$ can be split into the half-open intervals $P^{m}=\tau^{m} \cdot P^{0} \mathrm{~s}$,

$$
(0, \widetilde{\tau}]=\bigoplus_{m=0}^{\infty} P^{m},
$$

which, in their turn, are assumed to be split as in (6.11),

$$
P^{m}=P_{3}^{m} \oplus P_{2}^{m} \oplus P_{1}^{m} \oplus P_{0}^{m} .
$$

Lemma 6.1. Let $\mathcal{L}=\mathcal{L}(\beta, v, \gamma)$ be a one-dimensional quasilattice of level $m$, and let $x=\sigma\left(t^{\prime}\right)($ see $(3.9))$ be an arbitrary point in $\mathcal{L}$. Then the points neighboring to $x$ in $\mathcal{L}$ are $x+t_{-} \beta$ and $x+t_{+} \beta$. Here, for distinct sets $P^{m}\left(t_{-}^{\prime}, t_{+}^{\prime}\right)$ in the partition $P^{m}(|\gamma|)$, we obtain distinct local neighborhoods.

This follows from equation (6.9), the parametrization (3.9), and the isomorphism (6.10).

We say that a quasilattice $\mathcal{L}=\mathcal{L}(\beta, v, \gamma)$ has local type $j$, where $j=0,1,2,3$, if $|\gamma| \in P_{j}^{m}$. If $j=0,2$, the local types are degenerate, because arbitrarily small changes of the length $|\gamma|$ lead to the change of the local type of $\mathcal{L}$. We say that two quasilattices $\mathcal{L}_{1}$ and $\mathcal{L}_{2}$ are locally equivalent,

$$
\mathcal{L}_{1} \stackrel{l}{\sim} \mathcal{L}_{2},
$$

if they are of the same local type; i.e., there exists $j$ such that $\left|\gamma_{1}\right| \in P_{j}^{m_{1}}$ and $\left|\gamma_{2}\right| \in P_{j}^{m_{2}}$. This definition shows that locally equivalent quasilattices $\mathcal{L}_{1}$ and $\mathcal{L}_{2}$ are locally indistinguishable: up to similarity, their points have one and the same first local neighborhood. We note that $\mathcal{L} \stackrel{s}{\sim} \mathcal{M}$ implies $\mathcal{L} \stackrel{l}{\sim} \mathcal{M}$, but not vice versa.

Directly from Lemma 6.1 , we obtain a description of all possible local types of quasilattices.

Theorem 6.2. Every one-dimensional quasilattice $\mathcal{L}$ in the two-dimensional Fibonacci quasilattice $\mathcal{F}^{2}$ has local type $j$, where $j=0,1,2,3$. Conversely, every such local type is realized by some quasilattice $\mathcal{L}$ in $\mathcal{F}^{2}$.

We now use the results obtained above to the classification of the quasilattices $\mathcal{L}=$ $\mathcal{L}(\beta, v, \gamma)$ displayed in Figure 1.1. Using the equations of the corresponding lines $L$ (see Table 5.1), we find the lengths of the parameter intervals:

$$
\left|\gamma_{\mathcal{F}}\right|=\widetilde{\tau}, \quad\left|\gamma_{\mathcal{L}_{1}}\right|=1, \quad\left|\gamma_{\mathcal{L}_{2,0}}\right|=2 \tau, \quad\left|\gamma_{\mathcal{L}_{2}}\right|=\tau+\tau^{3}, \quad\left|\gamma_{\mathcal{L}_{3}}\right|=\widetilde{\tau}
$$

Combining this with (6.1), we see that the lattices in question form three distinct similarity classes,

$$
\mathcal{F} \stackrel{s}{\sim} \mathcal{L}_{1} \stackrel{s}{\sim} \mathcal{L}_{3}, \quad \mathcal{L}_{2,0} \stackrel{s}{\sim} \mathcal{L}_{2}
$$

Using (6.14) once again, we conclude, by decomposition (6.12), that the quasilattices $\mathcal{F}, \mathcal{L}_{1}$, and $\mathcal{L}_{3}$ have local type $j=0$, and the quasilattices $\mathcal{L}_{2,0}$ and $\mathcal{L}_{2}$ have local types 
$j=2$ and $j=1$, respectively; i.e., $\mathcal{L}_{2,0}$ and $\mathcal{L}_{2}$ are not locally equivalent, $\mathcal{L}_{2,0} \stackrel{l}{\nsim} \mathcal{L}_{2}$. Consequently, in the rough classification, the quasilattices in question again form three distinct local classes.

\section{$\S 7$. Appendix.}

The $\mathcal{F}_{1}$-ALgORIthm OF DIVISION IN THE FibonaCCI RING

7.1. To pass from a nonhomogeneous quasilattice $\mathcal{L}=\mathcal{L}(\beta, v, \gamma)$ to the corresponding homogeneous quasilattice $\mathcal{L}_{0}=\mathcal{L}_{0}\left(\beta, 0, \gamma_{0}\right)$ (see Subsection 3.3), we must know the shift vector $v$. For this, we must know how to find a particular solution $\left(x_{10}^{\prime}, x_{20}^{\prime}\right)$ of the nonhomogeneous equation

$$
a_{1}^{\prime} x_{1}^{\prime}+a_{2}^{\prime} x_{2}^{\prime}=c^{\prime}
$$

in $\mathcal{O}^{2}$.

The Fibonacci ring $\mathcal{O}$ is well known to be Euclidean with division algorithm with respect to the norm (see 11); therefore, particular solutions of (7.1) can be found by the Euclidean algorithm. We note that this algorithm is very time-consuming.

On the other hand, an application of the quasilattice $\mathcal{F}$ (see (1.3)) makes it possible to define a division algorithm in the Fibonacci ring $\mathcal{O}$ in the same way as in the case of the ring of rational integers $\mathbb{Z}$, thus obtaining a fast division algorithm in $\mathcal{O}$. The Euclidean algorithm arising in this way is a two-step algorithm (see [4), and it should be noted that, in essence, this algorithm is one-dimensional. In Theorem 7.1, we obtain an upper bound for the number of steps in this algorithm.

7.2. To construct this algorithm (the $\mathcal{F}_{1}$-algorithm), we use the modified quasilattice $\mathcal{F}_{1}=\tau \cdot \mathcal{F}$ obtained by $\tau$-inflation from the Fibonacci quasilattice $\mathcal{F}=\delta(\mathbb{Z})$, where $\delta(N)=N+[(N+1) \tau] \cdot \tau$ for $N \in \mathbb{Z}$. We have

$$
\mathcal{F}_{1}=\delta_{1}(\mathbb{Z}), \quad \text { where } \delta_{1}(\mathbb{Z})=[(N+1) \tau]+(N-[(N+1) \tau]) \cdot \tau .
$$

The replacement of the basic quasilattice $\mathcal{F}$ with the compressed quasilattice $\mathcal{F}_{1}$ is necessary, because the difference function

$$
\Delta \delta_{1}(N)=\delta_{1}(N)-\delta_{1}(N-1)=1 \text { or } \tau
$$

takes only positive values not exceeding 1 . This fact allows us to formally use the quasilattice $\mathcal{F}_{1}$ in the division algorithm instead of the ring of rational integers $\mathbb{Z}$.

Suppose $r_{0} \geq r_{1}>0$, where $r_{i} \in \mathcal{O}$. By (7.2), there exists a unique $N_{0} \in \mathbb{N}=$ $\{0,1,2, \ldots\}$ satisfying the conditions

$$
q\left(N_{0}\right) \leq \frac{r_{0}}{r_{1}}<q\left(N_{0}+1\right), \quad \text { where } q(N)=\delta_{1}(N) .
$$

Using (7.3), we obtain the representation

$$
\frac{r_{0}}{r_{1}}=q\left(N_{0}\right)+\frac{r_{2}}{r_{1}}, \quad \text { where } r_{2} \in \mathcal{O}, \quad r_{2} \geq 0, \text { and } 0 \leq \frac{r_{2}}{r_{1}}<1 \text {. }
$$

The $\mathcal{F}_{1}$-algorithm consists in recursive repetition of the action (7.4):

$$
\begin{gathered}
\frac{1}{\theta_{0}}=q\left(N_{0}\right)+\theta_{1}, \\
\frac{1}{\theta_{1}}=q\left(N_{1}\right)+\theta_{2}, \\
\ldots \ldots \ldots . \\
\frac{1}{\theta_{i-1}}=q\left(N_{i-1}\right)+\theta_{i}, \\
\frac{1}{\theta_{i}}=q\left(N_{i}\right)+\theta_{i+1}, \\
\ldots \ldots \ldots \ldots
\end{gathered}
$$

where $\theta_{i}=\frac{r_{i+1}}{r_{i}}, 0 \leq \theta_{i}<1, q\left(N_{i}\right) \geq 1$ for $i=0,1,2, \ldots$. 
7.3. We prove that the $\mathcal{F}_{1}$-algorithm (7.5) is finite, i.e., that $\theta_{n}=0$ for some $n \in \mathbb{N}$. Let $\theta_{i} \neq 0$. Then

$$
\theta_{i}=\frac{1}{q\left(N_{i}\right)+\theta_{i+1}}, \quad 0<\theta_{i}<\frac{1}{q\left(N_{i}\right)} .
$$

For $N_{i} \geq 4$, we obtain the inequalities

$$
\theta_{i}<\frac{1}{q(4)}=\frac{1}{3+\tau}, \quad\left|q^{\prime}\left(N_{i}\right)\right| \leq \widetilde{\tau},
$$

because if $\delta^{\prime}(N) \in(-1, \tau)$, then $\delta_{1}^{\prime}(N) \in(-\widetilde{\tau}) \cdot(-1, \tau)=(-1, \widetilde{\tau})$.

Similarly,

$$
\begin{array}{lll}
\theta_{i}<\frac{1}{q(3)}=\frac{1}{2+\tau}=\tau^{2}, & q^{\prime}\left(N_{i}\right)=q^{\prime}(3)=2-\widetilde{\tau}=\tau^{2} & \text { for } N_{i}=3 ; \\
\theta_{i}<\frac{1}{q(2)}=\frac{1}{1+\tau}=\tau, & q^{\prime}\left(N_{i}\right)=q^{\prime}(2)=1-\widetilde{\tau}=-\tau & \text { for } N_{i}=2 ; \\
\theta_{i}=\frac{1}{q(1)+\theta_{i+1}}=\frac{1}{1+\theta_{i+1}}, & q^{\prime}\left(N_{i}\right)=q^{\prime}(1)=1 & \text { for } N_{i}=1 .
\end{array}
$$

Thus, in (7.8), for $N_{i}=1$, we have an obstruction for proving that the $\mathcal{F}_{1}$-algorithm is finite. This means that (7.5) is not the usual Euclidean algorithm. Indeed, below we shall see that (7.5) is a two-step algorithm in the sense of Cooke [4]. For the proof, we need additional information about the quantity $\theta_{i+1}$ at the next step $N_{i+1}$.

If $N_{i+1}=2$, then, by (7.8),

$$
\theta_{i} \cdot \theta_{i+1}=\frac{\theta_{i+1}}{\theta_{i+1}+1}<\frac{\tau}{1+\tau}=\tau^{2},
$$

because $0 \leq \theta_{i+1}<\tau$. Otherwise, by (7.6), we would have $\frac{1}{\theta_{i}}=q\left(N_{i}\right)+\theta_{i+1}>1+\tau=$ $q(2)$, or $q(2)<\frac{1}{\theta_{i}}$, which implies that $N_{i} \geq 2$, contrary to the assumption $N_{i}=1$. Therefore, for $N_{i+1}=2$, we have (see Table 7.1)

$$
\theta_{i} \cdot \theta_{i+1}<\tau^{2}, \quad q^{\prime}\left(N_{i}\right) \cdot q^{\prime}\left(N_{i+1}\right)=-\tau .
$$

TABLE 7.1

\begin{tabular}{|c|c|c|c|c|c|}
\hline$N$ & 1 & 2 & 3 & 4 & 5 \\
\hline$\delta_{1}(N)$ & 1 & $1+\tau$ & $2+\tau$ & $3+\tau$ & $3+2 \tau$ \\
$\delta_{1}^{\prime}(N)$ & 1 & $1-\widetilde{\tau}$ & $2-\widetilde{\tau}$ & $3-\widetilde{\tau}$ & $3-\widetilde{2 \tau}$ \\
\hline
\end{tabular}

Let $N_{i+1}=3$. Then, using (7.8) once again, we arrive at the inequality

$$
\theta_{i+1}=\frac{1}{q\left(N_{i+1}\right)+\theta_{i+2}}<\frac{1}{q\left(N_{i+1}\right)}=\frac{1}{q\left(N_{3}\right)}=\tau^{2} .
$$

Combining this with (7.8) for $N_{i+1}=3$, we obtain

$$
\theta_{i} \cdot \theta_{i+1}=\frac{\theta_{i+1}}{1+\theta_{i+1}}<\frac{1}{3+\tau}, \quad q^{\prime}\left(N_{i}\right) \cdot q^{\prime}\left(N_{i+1}\right)=\tau^{2} .
$$

Similarly, in the remaining cases $\left(N_{i+1} \geq 4\right)$ we have

$$
\theta_{i+1}<\frac{1}{q\left(N_{i+1}\right)} \leq \frac{1}{q\left(N_{4}\right)}=\frac{1}{3+\tau}
$$

whence

$$
\theta_{i} \cdot \theta_{i+1}=\frac{\theta_{i+1}}{1+\theta_{i+1}}<\frac{1}{4+\tau}, \quad q^{\prime}\left(N_{i}\right)=1, \quad\left|q^{\prime}\left(N_{i+1}\right)\right| \leq \widetilde{\tau} .
$$


Since $\frac{r_{i+1}}{r_{i}}=\theta_{i}$, we can represent the $\mathcal{F}_{1}$-algorithm (7.5) in a more conventional form:

$$
\begin{gathered}
r_{0}=q\left(N_{0}\right) r_{1}+r_{2}, \\
r_{1}=q\left(N_{1}\right) r_{2}+r_{3}, \\
\cdots \cdots \cdots \\
r_{i-1}=q\left(N_{i-1}\right) r_{i}+r_{i+1}, \\
r_{i}=q\left(N_{i}\right) r_{i+1}+r_{i+2},
\end{gathered}
$$

Now, we estimate the quantity $\left|N_{F / \mathbb{Q}}\left(r_{i+1}\right)\right|$ from above. Applying the equation $r_{i+1}=$ $\theta_{i} r_{i}$ several times, we obtain the following relation between the remainders $r_{i+1}$ and $r_{0}$ for all $i \geq 0$ :

$$
r_{i+1}=\theta_{i} \cdots \theta_{0} r_{0}
$$

For our purposes, it is convenient to represent (17.14) in a matrix form. Since $r_{i+2}=$ $-q\left(N_{i}\right) r_{i+1}+r_{i}$, we have the matrix relation

$$
\left(r_{i+1}, r_{i+2}\right)=\left(r_{0}, r_{1}\right) \cdot M_{i},
$$

where $M_{i}=Q_{0} \cdots Q_{i}$ and the matrices $Q_{j}$ are of the form $Q_{j}=\left(\begin{array}{l}0 \\ 1-q\left(N_{j}\right)\end{array}\right)$. Let $\left|\left(r_{i+1}, r_{i+2}\right)\right|$ denote the length of the vector $\left(r_{i+1}, r_{i+2}\right)$. Then, obviously,

$$
\left|r_{i+1}^{\prime}\right| \leq\left|\left(r_{i+1}^{\prime}, r_{i+2}^{\prime}\right)\right|
$$

and the right-hand side can be estimated by (7.15) as follows:

$$
\left|\left(r_{i+1}^{\prime}, r_{i+2}^{\prime}\right)\right|=\left|\left(r_{0}^{\prime}, r_{1}^{\prime}\right) \cdot M_{i}^{\prime}\right| \leq\left|\left(r_{0}^{\prime}, r_{1}^{\prime}\right) R_{0} \cdots R_{i}\right|,
$$

where

$$
R_{j}=\left(\begin{array}{cc}
0 & 1 \\
1 & \left|q^{\prime}\left(N_{j}\right)\right|
\end{array}\right) .
$$

Let $v$ be a vector in $\mathbb{R}^{2}$, let $R=\left(\begin{array}{ll}0 & 1 \\ 1 & r\end{array}\right)$ with $r \geq 0$, and let $(\cdot, \cdot)$ be the scalar product in $\mathbb{R}^{2}$. We have $|v \cdot R|^{2}=(v \cdot R, v \cdot R)=\left(v \cdot R R^{t}, v\right) \leq \Lambda \cdot(v, v)$, where $\Lambda$ is the maximal eigenvalue of the matrix $R R^{t}$. We use the inequality

$$
|v \cdot R|^{2} \leq \Lambda \cdot|v|^{2}
$$

which can be checked as follows: $|v \cdot R|^{2}=(v \cdot R, v \cdot R)=\left(v \cdot R R^{t}, v\right) \leq \Lambda \cdot(v, v)$, where $\Lambda$ is the maximal eigenvalue of the matrix $R R^{t}$.

Since $R=R^{t}$ is a symmetric matrix, we have

$$
\Lambda=\lambda^{2}
$$

where $\lambda=\frac{r+\sqrt{r^{2}+4}}{2}$ is the maximal eigenvalue of the matrix $R$. Now, from (7.19) and (7.20), we deduce the required inequality

$$
|v \cdot R| \leq \lambda|v|
$$

Applying this to the right-hand side of (7.17), we obtain

$$
\left|\left(\left|r_{0}^{\prime}\right|,\left|r_{1}^{\prime}\right|\right) R_{0} \cdots R_{i}\right| \leq \lambda_{0} \cdots \lambda_{i}\left|\left(\left|r_{0}^{\prime}\right|,\left|r_{1}^{\prime}\right|\right)\right|=\lambda_{0} \cdots \lambda_{i}\left|\left(r_{0}^{\prime}, r_{1}^{\prime}\right)\right|,
$$

where

$$
\lambda_{j}=\frac{\left|q^{\prime}\left(N_{j}\right)\right|+\sqrt{q^{\prime}\left(N_{j}\right)^{2}+4}}{2}
$$

is an eigenvalue of the matrix $R_{j}$ (see (7.18)). Now, using (7.16), we get the inequality $\left|r_{i+1}^{\prime}\right| \leq \lambda_{0} \cdots \lambda_{i}\left|\left(r_{0}^{\prime}, r_{1}^{\prime}\right)\right|$. Recalling (7.14), we arrive at an inequality for the norms of the remainders $r_{i+1}$ in the $\mathcal{F}_{1}$-algorithm (7.13):

$$
\left|N_{F / \mathbb{Q}}\left(r_{i+1}\right)\right| \leq\left(\theta_{0} \lambda_{0}\right) \cdots\left(\theta_{i} \lambda_{i}\right) \cdot\left|r_{0}\right| \cdot\left|\left(r_{0}^{\prime}, r_{1}^{\prime}\right)\right| .
$$


By (7.7), (7.8), and (7.22), we have the following inequalities for the products $\theta_{i} \lambda_{i}$ :

$$
\begin{array}{ll}
\theta_{i} \lambda_{i} \leq \tau \cdot \frac{\tau+\sqrt{\tau^{2}+4}}{2}<0.838 & \text { for } N_{i}=2 \\
\theta_{i} \lambda_{i} \leq \tau^{2} \cdot \frac{\tau^{2}+\sqrt{\tau^{4}+4}}{2}<0.46 & \text { for } N_{i}=3 \\
\theta_{i} \lambda_{i} \leq \frac{1}{3+\tau} \cdot \frac{\widetilde{\tau}+\sqrt{\tau^{2}+4}}{2}<0.6 & \text { for } N_{i} \geq 4
\end{array}
$$

Now, we use (7.7) and (7.8) to obtain upper bounds for the pairwise products $\left(\theta_{i} \lambda_{i}\right)$. $\left(\theta_{i+1} \lambda_{i+1}\right)$, representing them in the form $\left(\theta_{i} \theta_{i+1}\right) \cdot\left(\lambda_{i} \lambda_{i+1}\right)$ :

$$
\begin{array}{lll}
\left(\theta_{i} \theta_{i+1}\right) \cdot\left(\lambda_{i} \lambda_{i+1}\right) \leq \tau^{2} \cdot \frac{1+\sqrt{5}}{2} \cdot \frac{\tau+\sqrt{\tau^{2}+4}}{2}<0.844 & \text { for } N_{i}=1, & N_{i+1}=2 ; \\
\left(\theta_{i} \theta_{i+1}\right) \cdot\left(\lambda_{i} \lambda_{i+1}\right) \leq \frac{1}{3+\tau} \cdot \widetilde{\tau} \cdot \frac{\tau^{2}+\sqrt{\tau^{4}+4}}{2}<0.838 & \text { for } N_{i}=1, & N_{i+1}=3 ; \\
\left(\theta_{i} \theta_{i+1}\right) \cdot\left(\lambda_{i} \lambda_{i+1}\right) \leq \frac{1}{4+\tau} \cdot \widetilde{\tau} \cdot \frac{\widetilde{\tau}+\sqrt{\tau^{2}+4}}{2}<0.74 & \text { for } N_{i}=1, & N_{i+1} \geq 4 .
\end{array}
$$

We split the product $\left(\theta_{0} \lambda_{0}\right) \cdots\left(\theta_{i} \lambda_{i}\right)$ on the right-hand side of inequality (7.23) into: 1) the double pairs $\left(\theta_{j} \lambda_{j}\right)\left(\theta_{j+1} \lambda_{j+1}\right)$ for $N_{j}=1$; and 2) the single pairs $\left(\theta_{k} \lambda_{k}\right)$ that do not appear in 1) for $N_{k} \geq 2$. We estimate these pairs with the help of (7.24) and (7.25) and apply the result to (7.23). We obtain

$$
\begin{array}{ll}
\left|N_{F / \mathbb{Q}}\left(r_{i+1}\right)\right| \leq 0.844^{i / 2} \cdot\left|r_{0}\right| \cdot\left|\left(r_{0}^{\prime}, r_{1}^{\prime}\right)\right| & \text { if } i \text { is even, } \\
\left|N_{F / \mathbb{Q}}\left(r_{i+1}\right)\right| \leq 0.844^{(i+1) / 2} \cdot\left|r_{0}\right| \cdot\left|\left(r_{0}^{\prime}, r_{1}^{\prime}\right)\right| & \text { if } i \text { is odd. }
\end{array}
$$

Now, we observe that, in the $\mathcal{F}_{1}$-algorithm (17.13), the remainders $r_{i+1}$ belong to the ring $\mathcal{O}$. Therefore, $\left|N_{F / \mathbb{Q}}\left(r_{i+1}\right)\right| \geq 1$ if $r_{i+1} \neq 0$. This fact and inequalities (17.26) lead to the following statement.

Theorem 7.1. For all $x$ and $y(x \geq y>0)$ in the Fibonacci ring $\mathcal{O}=\mathbb{Z}[\tau]$, the $\mathcal{F}_{1}$-algorithm (7.13) terminates after at most

$$
12\left(\ln (x)+\ln \left(\left|\left(x^{\prime}, y^{\prime}\right)\right|\right)\right)+1
$$

steps.

Remark 7.1. Numerical calculations show that, as a rule, the factor 12 in (7.27) can be replaced by 1 .

\section{REFERENCES}

[1] Z. I. Borevich and I. R. Shafarevich, The theory of numbers, 3rd ed., "Nauka", Moscow, 1985; English transl. of 1st ed., Pure Appl. Math., vol. 20, Acad. Press, New York-London, 1966. MR0816135 (88f:11001) MR0195803 (33:4001)

[2] V. G. Zhuravlev, One-dimensional Fibonacci partitions, Proc. 17th Internat. Summer Workshop on Modern Problems of Theoretical and Mathematical Physics, Kazan', 2005, pp. 40-55. (Russian)

[3] - Sums of squares over the Fibonacci o-ring, Zap. Nauchn. Sem. S.-Peterburg. Otdel. Mat. Inst. Steklov. (POMI) 337 (2006), 165-190; English transl., J. Math. Sci. (N.Y.) 143 (2007), no. 3, 3108-3123. MR2271962 (2007i:11030)

[4] G. Cooke, A weakening of the Euclidean property for integral domains and applications to algebraic number theory. I, J. Reine Angew. Math. 282 (1976), 133-156. MR0406973 (53:10758a)

[5] N. P. Fogg, Substitutions in dynamics, arithmetics and combinatorics, Lecture Notes in Math., vol. 1794, Springer-Verlag, Berlin, 2002. MR1970385 (2004c:37005)

[6] D. E. Knuth, Fibonacci multiplication, Appl. Math. Lett. 1 (1988), 57-60. MR0947168 (89f:11031)

[7] R. Lifshitz, The square Fibonacci tiling, J. Alloys Compounds 342 (2002), 186-190.

[8] Yu. V. Matiyasevich, The connection between Hilbert's tenth problem and systems of equations between words and lengths, Zap. Nauchn. Sem. Leningrad. Otdel. Mat. Inst. Steklov. (LOMI) 8 (1968), 132-144; English transl., Sem. in Math. Steklov Math. Inst., Leningrad 8 (1970), 61-67. MR 0246772(40:41) 
[9] - Two reductions of Hilbert's tenth problem, Zap. Nauchn. Sem. Leningrad. Otdel. Mat. Inst. Steklov. (LOMI) 8 (1968), 145-158; English transl., Sem. in Math. Steklov Math. Inst., Leningrad 8 (1970), 68-74. MR0246773 (40:42)

[10] R. V. Moody, Model sets: A survey, Quasicrystals to More Complex Systems (F. Alex, F. Dénoyer, and J. P. Gazeau, eds.), EPD Science, Les Ulis, and Springer-Verlag, Berlin, 2000, pp. 145-166.

Vladimir State Pedagogical University, Prospekt Stroitelei 11, 600024, Vladimir, Russia E-mail address: vzhuravlev@mail.ru

Received 11/SEP/2006

Translated by B. M. BEKKER 